\title{
Encoding of Tactile Stimulus Location by Somatosensory Thalamocortical Ensembles
}

\author{
Asif A. Ghazanfar, Christopher R. Stambaugh, and Miguel A. L. Nicolelis \\ Department of Neurobiology, Duke University Medical Center, Durham, North Carolina 27710
}

The exquisite modular anatomy of the rat somatosensory system makes it an excellent model to test the potential coding strategies used to discriminate the location of a tactile stimulus. Here, we investigated how ensembles of simultaneously recorded single neurons in layer $\mathrm{V}$ of primary somatosensory (SI) cortex and in the ventral posterior medial (VPM) nucleus of the thalamus of the anesthetized rat may encode the location of a single whisker stimulus on a single trial basis. An artificial neural network based on a learning vector quantization algorithm, was used to identify putative coding mechanisms. Our data suggest that these neural ensembles may rely on a distributed coding scheme to represent the location of single whisker stimuli. Within this scheme, the temporal modulation of neural ensemble firing rate, as well as the temporal interactions between neurons, contributed significantly to the representation of stimulus location. The relative contribution of these temporal codes increased with the number of whiskers that the ensembles must discriminate among. Our results also indicated that the SI cortex and the VPM nucleus may function as a single entity to encode stimulus location. Overall, our data suggest that the representation of somatosensory features in the rat trigeminal system may arise from the interactions of neurons within and between the SI cortex and VPM nucleus. Furthermore, multiple coding strategies may be used simultaneously to represent the location of tactile stimuli.

Key words: population coding; multi-electrode; temporal code; ventral posterior medial nucleus; barrel cortex; primary somatosensory cortex
Rodents actively use their facial whiskers to explore their environment. Removal of these whiskers results in impaired performance on various tactile discrimination tasks (Vincent, 1912; Schiffman et al., 1970; Brecht et al., 1997). These behavioral experiments have underscored the importance of the facial whiskers in determining the spatial location of tactile stimuli. For example, by clipping the large caudal vibrissae of the rat's whisker pad and comparing performance of rats on spatial versus object recognition tasks, Brecht et al. (1997) demonstrated that these large caudal whiskers were critically involved in spatial tasks but not in object recognition tasks. These authors (Brecht et al., 1997) suggested that these whiskers act as "distance decoders," the function of which is to determine the location of obstacles and openings.

At the neural level, experimental lesions within the "whisker area" of the rat somatosensory system support the hypothesis that the caudal whiskers and their associated neural pathways are necessary for spatial discrimination (Hutson and Masterton, 1986). It is unclear, however, how neurons may encode the spatial location of tactile stimuli. Coding mechanisms for determining the spatial location of a stimulus in sensory space generally fall into two categories: local versus distributed coding. In the local

Received Nov. 24, 1999; revised March 6, 2000; accepted March 6, 2000.

This work was supported by the Whitehall Foundation, the Klingenstein Foundation, the Whitehead Foundation, and the National Institute for Dental Research (DE-11121-01). We thank Peter Cariani, Mark Laubach, and Marshall Shuler for sharing their insights on neural coding and for their helpful comments on this manuscript. We also thank an anonymous reviewer for helpful comments and suggestions.

Correspondence should be addressed to Dr. Miguel Nicolelis, Department of Neurobiology, Box 3209, Duke University Medical Center, Durham, NC 27710. E-mail: nicoleli@neuro.duke.edu.

Dr. Ghazanfar's present address: Primate Cognitive Neuroscience Lab, Department of Psychology, 33 Kirkland Street, Room 984, Harvard University, Cambridge, MA 02138. E-mail: aghazanf@wjh.harvard.edu.

Copyright (C) 2000 Society for Neuroscience $0270-6474 / 00 / 203761-15 \$ 15.00 / 0$ coding scheme, the sensory space is divided into nonoverlapping areas that can be resolved by small groups of topographically arranged neurons. These neurons necessarily have small receptive fields. One of the potential benefits of topographic maps in sensory systems is the ability to easily identify the location of a stimulus: localized groups of neurons respond specifically to the presence of a stimulus in a restricted portion of the sensory space, whereas the other neurons are quiescent. Thus, local coding can offer exquisite specificity and speed in behavioral response. However, lesions of a particular region of this map would render the system unable to identify stimuli delivered to discrete locations on the receptor organ. Conversely, in the distributed coding scheme, neurons have relatively large and overlapping receptive fields compared with the sensory resolution measured behaviorally. Distributed representations allow neurons to be computationally flexible (neurons can participate in many different aspects of sensory processing) (Richmond and Optican, 1987; Victor and Purpura, 1996) and resistant to both central and peripheral injury (Nicolelis, 1997).

The specialized structure of the rodent somatosensory pathway is well suited to testing which of these potential coding strategies, local versus distributed, is used to identify the spatial location of sensory stimuli. The rat trigeminal somatosensory pathway consists of topographically arranged clusters (or modules) of neurons: "barrel columns" in the cortex (Woolsey and Van der Loos, 1970; Killackey, 1973), "barreloids" in the thalamus (Van der Loos, 1976), and "barrelettes" in the brainstem (Ma, 1991). Each module corresponds isomorphically to a single whisker on the snout. The modular and topographic anatomy suggests that this system may use a local coding scheme, whereby each module encodes the spatial location of a single caudal whisker (Nelson and Bower, 1990). However, the discrete cytoarchitecture of this pathway stands in contrast to what is known regarding the phys- 
iology of this system. Neurons in these structures have large receptive fields that extend well beyond a single caudal whisker (Simons, 1978; Chapin, 1986; Armstrong-James and Fox, 1987; Simons and Carvell, 1989; Nicolelis and Chapin, 1994; Moore and Nelson, 1998; Zhu and Connors, 1999; Ghazanfar and Nicolelis, 1999). In addition, functional studies have shown in primary somatosensory (SI) cortex (Kleinfeld and Delaney, 1996; Masino and Frostig, 1996; Peterson et al., 1998; Sheth et al., 1998), ventral posterior medial (VPM) nucleus (Nicolelis and Chapin, 1994; Ghazanfar and Nicolelis, 1997), and SpV (Nicolelis et al., 1995) that stimulation of individual whiskers results in responses that extend well beyond a single barrel cortical column or VPM nucleus barreloid or spinal trigeminal nucleus $(\mathrm{SpV})$ barrelette.

To date, no study has explored the potential coding strategies in an anatomically modular and topographic sensory system used to represent the location of a stimulus on a single trial basis. The presumption has always been that such systems use "local" coding schemes to encode stimulus location. As a first step to address this issue, we tested the potential coding strategies of simultaneously recorded ensembles of single neurons distributed across layer $\mathrm{V}$ of SI cortex and VPM nucleus of the thalamus in the anesthetized rat and investigated how these two structures may interact with each other to encode the location of simple tactile stimuli.

\section{MATERIALS AND METHODS}

\section{Animals and surgical procedures}

Nine adult female Long-Evans rats (250-300 gm) were used in these experiments. Details of surgical procedures have been described elsewhere (Nicolelis et al., 1997a). Briefly, animals were anesthetized with intraperitoneal injections of sodium pentobarbital (Nembutal, $50 \mathrm{mg} / \mathrm{kg}$ ) and transferred to a stereotaxic apparatus. When necessary, small supplementary injections of sodium pentobarbital $(\sim 0.1 \mathrm{cc})$ were administered to maintain anesthesia during the surgery. After retraction of the skin and soft tissue, small, rectangular craniotomies were made over the SI barrel cortex and/or the VPM nucleus of the thalamus using stereotaxic coordinates. For layer V cortical implants, stainless-steel microwire arrays (NB Labs, Dennison, TX) consisting of two rows, separated by 1 $\mathrm{mm}$, of eight microwires were used. Each microwire was Teflon-coated and had a $50 \mu \mathrm{m}$ tip diameter. The inter-microwire distance within a row was $200 \mu \mathrm{m}$. For V PM nucleus implants, two bundles of eight microwires, cut at two different lengths, were used. The distance between bundles was $\sim 1 \mathrm{~mm}$. For all animals, we successfully targeted the representation for the large, caudal whiskers (B1-4, C1-4, D1-4, and E1-4) [see Ghazanfar and Nicolelis (1999) for details on identifying target locations]. On proper placement, microwire implants were cemented to the animal's skull with dental acrylic. The location of all microwires was assessed by qualitative receptive field mapping during surgical implantation, later confirmed by the quantitative response profiles of neurons, and then postmortem by light microscopic analysis of Nissl-stained sections.

\section{Data acquisition}

\section{Spike sorting}

After a recovery period of 5-7 d, animals were anesthetized with sodium pentobarbital $(50 \mathrm{mg} / \mathrm{kg})$ and transferred to a recording chamber where all experiments were performed. A head stage was used to connect the chronically implanted microwires to a preamplifier whose outputs were sent to a Multi-Neuronal Acquisition Processor (Plexon Inc., Dallas, TX) for on-line multi-channel spike sorting and acquisition (sampling rate $=$ $40 \mathrm{kHz}$ per channel). A maximum of four extracellular single units per microwire could be discriminated in real time using time-voltage windows and a principal component-based spike sorting algorithm (Abeles and Goldstein, 1977; Nicolelis and Chapin, 1994). Previous studies have revealed that under our experimental conditions, $\sim 80 \%$ of the microwires yield stable single units and an average of 2.3 single units can be well discriminated per microwire (Nicolelis et al., 1997a). Examples of waveforms and further details regarding acquisition hardware and spike sorting can be found elsewhere (Nicolelis and Chapin, 1994; Nicolelis et al., 1997a).

\section{Recording session and whisker stimulation}

After spike sorting, the simultaneous extracellular activity of all well isolated single units was recorded throughout the duration of all stimulation experiments. A computer-controlled vibromechanical probe was used to deliver innocuous mechanical stimulation to single whiskers on the mystacial pad contralateral to the microwire array implant. The independent stimulation of 16 whiskers was performed per recording session per animal. Three hundred sixty trials were obtained per stimulated whisker, and the probe was then moved to another whisker (in random order). Whiskers were stimulated by positioning the probe just beneath an individual whisker, $\sim 5-10 \mathrm{~mm}$ away from the skin. Extreme care was taken to ensure that only a single whisker was being stimulated at all times. A step-pulse (100 msec in duration) delivered at $1 \mathrm{~Hz}$ by a Grass 8800 stimulator was used to drive the vibromechanical probe. The output of the stimulator was calibrated to produce a $\sim 0.5 \mathrm{~mm}$ upward deflection of whiskers. Stable levels of anesthesia were maintained by small supplemental injections of pentobarbital $(\sim 0.05 \mathrm{cc})$ and monitored through regular inspection of brain activity, breathing rates, and tailpinch responses.

\section{Data analysis}

\section{Firing rate and minimal latencies}

The minimal spike latency and the average evoked firing rate of each neuron were estimated using poststimulus time histograms (PSTHs) and cumulative frequency histograms (CFHs). CFHs were used to measure the statistical significance of sensory responses to tactile stimuli. These histograms depict the cumulative poststimulus deviations from prestimulus average firing seen in the PSTHs. In other words, the CFHs describe the probability that the cumulative frequency distribution in the histogram differs from a random distribution, as computed by a one-way Kolmogorov-Smirnov test. Neuronal responses were considered statistically significant if the corresponding CFH indicated a $p<0.01$. These analyses were performed on commercially available software (Stranger, Biographics, Winston-Salem, NC). For CFHs of statistically significant responses, the minimal latencies were measured using a single neuron analysis program based on Kernel Density Estimation and written in Matlab (Mathworks, Natick, MA) by Mark Laubach and Marshall Shuler (MacPherson and Aldridge, 1979; Richmond and Optican, 1987; Ghazanfar and Nicolelis, 1999). Details of this analysis procedure have been reported elsewhere (Ghazanfar and Nicolelis, 1999).

\section{Population histograms}

Population histograms describe the sensory response of simultaneously recorded neural ensemble to the deflection of a single whisker as a function of poststimulus time. These three-dimensional plots are essentially a collection of single neuron PSTHs stacked next to each other. These can be generated using a range of bin widths $(1,3,6,10,20$, and $40 \mathrm{msec}$ ). The $x$-axis of these plots represents poststimulus time in milliseconds, the $y$-axis represents the neuron number, and the $z$-axis represents response magnitude in spikes per second. The neurons are arranged randomly along the $y$-axis.

\section{Single trial analysis of neural ensemble firing patterns}

Extracting information from the firing patterns of populations of neurons is difficult largely because of the combinatorial complexity of the problem and the uncertainty about how information is encoded in the nervous system. Our previous studies indicated that a large number of neurons are active in the rat thalamocortical loop after the deflection of a single whisker (Nicolelis and Chapin, 1994; Ghazanfar and Nicolelis, 1997; Nicolelis et al., 1997a). At spike-to-spike resolution, there is also a high degree of variability in the spike train of an individual neuron (Shadlen and Newsome, 1998; A. Ghazanfar and M. Nicolelis, unpublished observations). Although both the number of spikes produced by a neuron and their timing may vary from trial to trial, at the neural ensemble level the location of a stimulus may be identified in a statistically predictable manner. Pattern recognition approaches using multivariate statistical methods, such as linear discriminant analysis, and artificial neural networks (ANNs) are effective tools for investigating this possibility (Deadwyler and Hampson, 1997; Nicolelis et al., 1999). 


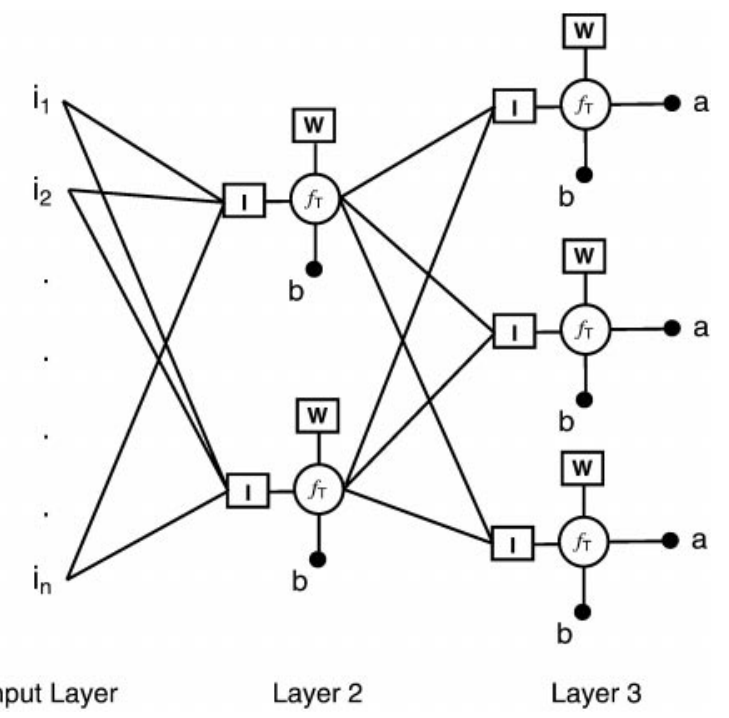

Figure 1. Statistical pattern recognition using an artificial neural network (ANN). The ANN used a nearest-neighbor classifier algorithm, learning vector quantization, to classify our large, sparse neural ensemble datasets. The system was a multilayered, feedforward ANN with full connectivity. In this case, the transfer function $\left(F_{\mathrm{t}}\right)$ is a Euclidean distance measure. The first layer consisted of our raw data; the second layer contained two artificial neural units (ANUs) for each class (i.e., the number of whiskers); the third layer had the same number of ANUs as classes; and the fourth layer (data not shown) was the output layer.

\section{Artificial neural network based on the learning vector quantization classifier}

In this study, an ANN was used for statistical pattern recognition analysis of the thalamocortical responses to tactile stimuli on a single trial basis. The ANN was constructed in Matlab using an optimized learning vector quantization (LVQ) algorithm (Kohonen, 1997). The LVQ ANN is a nearest-neighbor classifier, which provides a nonparametric technique for classifying large and sparse nonlinear pattern vectors. The LVQ algorithm was selected because of its simplicity of design and its ability to handle our extremely large and sparse neural ensemble data sets. By using this approach, preprocessing of neural ensemble data, by using principal or independent component analyses, was not necessary as a primary step in the analysis (Nicolelis et al., 1998). Thus, the only parameters available to the LVQ ANN for pattern recognition were the firing rate and the temporal patterning of neuronal firing within simultaneously recorded thalamocortical ensembles.

In the Appendix, we have described in detail the logical structure and mathematical basis for an LVQ algorithm-based ANN used in our study. Here, we will briefly review the specific parameters used in our study. The first layer defined the input layer and consisted of our raw spike train data (Fig. 1). The second layer contained two artificial neural units (ANUs) for each class (i.e., the number of stimulus locations-whiskers-to be discriminated). The output value of each second layer ANU was determined by an Euclidean distance function. The third layer of the ANN had the same number of units as the number of classes (i.e., the number of stimulus locations). A value of 1 was assigned to the third layer ANUs corresponding to the "winning" second layer ANU, whereas the rest of the third layer units were assigned the value of 0 . Thus, if the fifth ANU in the second layer had the greatest output value, the fifth ANU of the third layer would output 1 , whereas the rest of the ANUs in that layer would output $0 \mathrm{sec}$. Each ANU in this final layer represented a unique subset of second layer ANUs. The fourth and final (output) layer of the ANN also contained the same number of units as there were classes of stimulus sites to be discriminated.

The analysis of our data set included two phases: training and testing. During the training phase of the analysis, the ANN searched for patterns closest in Euclidean distance to one of the weight vectors. For every analysis in this study, $25 \%$ of the trials were used for training the LVQ ANN, and the remaining $75 \%$ were used as testing trials. To obtain an accurate assessment of the network performance in classifying our neu- ral ensemble data, four-way cross-validation was used and provided us with a measurement of error. Thus, all trials used for training in one session were then used in an independent session for testing and vice versa. The spike train of each neuron in the ensemble from 0 to $40 \mathrm{msec}$ poststimulus time was used in all analyses. Unless noted otherwise, 4 msec bins were used to define the contribution of each neuron to the ensemble input vector.

\section{Exploring putative coding mechanisms}

Our basic approach to investigating coding mechanisms was to compare statistical pattern recognition performance by the ANN using normal, "raw" neural ensemble data versus different manipulations of that data set. Raw data manipulations included removing the number of neurons within an ensemble, reducing the temporal resolution of spike trains, and disrupting the phase relationships or correlated activity between neurons.

Local versus distributed coding. One of the hallmarks of distributed coding is the graceful degradation of ensemble performance after the removal of neurons from an ensemble. We tested this by measuring ensemble performance on discriminating four different whiskers (B1, B4, $\mathrm{E} 1$, and $\mathrm{E} 4$; chance performance $=25 \%$ ) and then removing the best predictor neuron from the ensemble one at a time, sequentially. The best predictor neuron was determined by running the analysis with each neuron taken out in turn and then finding the neuron that had the most detrimental effect on ensemble performance when removed. This neuron was then defined as the "best predictor" neuron of the ensemble. Once this neuron was removed, the analysis was run again to quantify the performance of the ensemble without that neuron and find the next best predictor neuron.

Because we found that the effect of removing neurons from ensembles resulted in a smooth degradation of ensemble performance (see Results), we were able to estimate the number of neurons needed to achieve a $99 \%$ correct level of performance by using a power function (Carpenter et al., 1999): $x=\log _{10}(z) / \log _{10}(y), \mathrm{x}^{*} \mathrm{w}=$ number of neurons needed, where $z$ equals the desired residual information ( 0.01 , for $99 \%$ correct) and $y$ equals the obtained residual for $w$ neurons actually recorded. A power function is necessary because information capacity changes nonlinearly with an increasing number of neurons.

Temporal structure of ensemble firing rate. To determine whether the temporal modulation of ensemble firing rates contributed to the performance of thalamocortical ensembles, the integration time used to describe the sensory response (i.e., bin size) of each neuron was systematically varied between 1 and $40 \mathrm{msec}$. Increasing the bin size degrades the temporal resolution of the response, allowing an assessment of the relative contributions of rate and temporal coding to ensemble performance.

Correlated activity across neuronal spike trains: spike-shifting testing trials. To explore the role of covariance structure on ensemble performance, linear discriminant analysis (LDA) (Tabachnick and Fidell, 1996) was used in our analyses. LDA was used to identify sources of variance and to measure the covariance of firing rate activity among simultaneously recorded neurons. Such sources of covariance have been suggested to be important for the coding of sensory stimuli (Nicolelis et al., 1997b) and behavioral events (Deadwyler et al., 1996). Another useful characteristic of LDA is that it derives classification functions for trialby-trial discrimination between different experimental groups (in this case, the different stimulation sites). This statistical technique has been used extensively for neural ensemble data analysis (Gochin et al., 1994; Schoenbaum and Eichenbaum, 1995; Deadwyler et al., 1996; Nicolelis et al., 1997b). Our application of LDA to somatosensory neural data sets has been described in detail elsewhere (Nicolelis et al., 1997b).

To apply LDA and test the role of correlated activity among neuronal firing patterns in the performance of our ensembles, raw spike trains within an ensemble were temporally shifted in one of two ways. In the first method, "spike shifting," the spike trains within ensemble responses were shifted relative to each other in random order, between \pm 6 and 12 msec. Thus, from a single trial's ensemble response, the spike train of neuron 1 may have been shifted $+7 \mathrm{msec}$, that of neuron 2 may have been shifted $-10 \mathrm{msec}$, so on and so forth. This was done for every trial in the testing phases of the analyses. The range of "shift" times was selected based on the finding that neural ensemble performance significantly degraded only with bin sizes $>6 \mathrm{msec}$ for both SI cortex and VPM nucleus (see Results, Fig. 5B). The second method, "trial-shuffling," was used to randomly replace the spike trains of each neuron with those from another trial. After either of these procedures, normal and shifted 


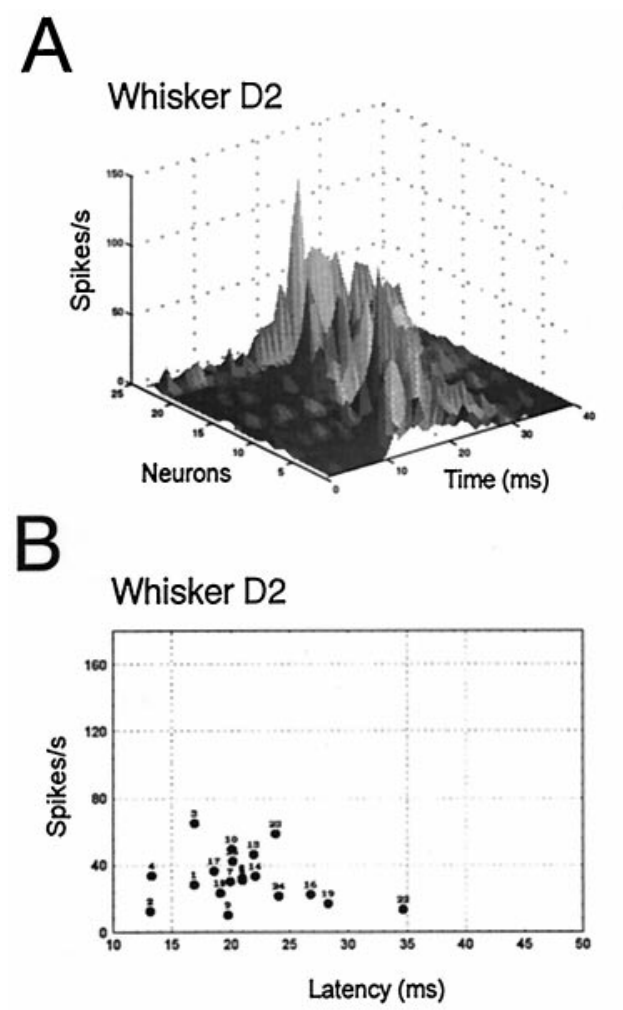

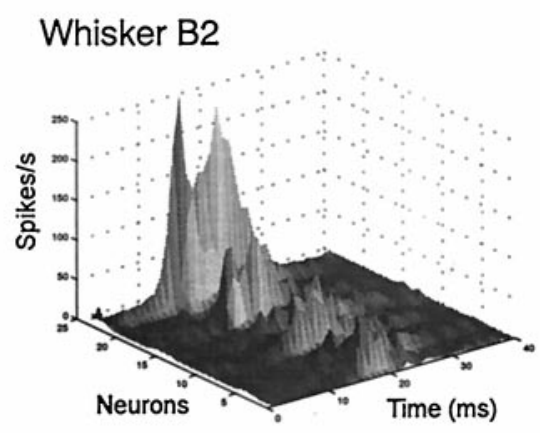

\section{Whisker B2}

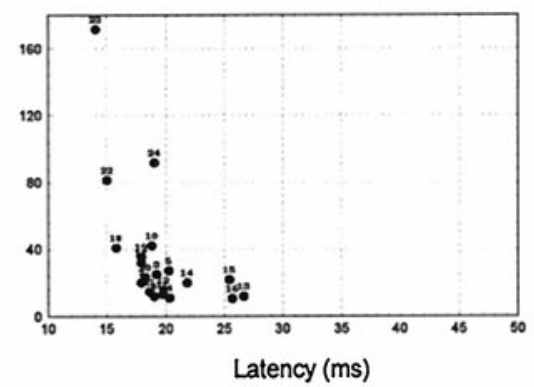

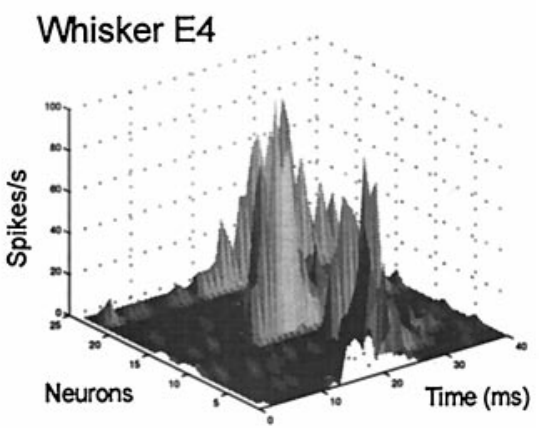

Whisker E4

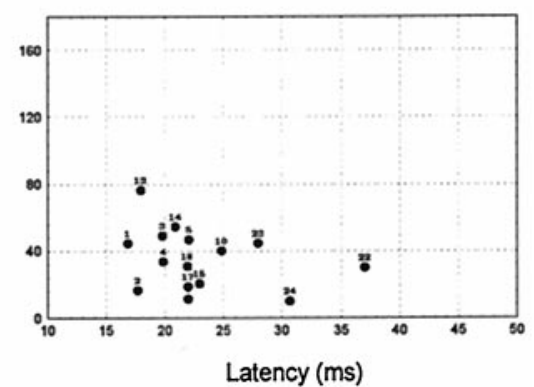

Figure 2. The spatiotemporal responses of the same ensemble to different whiskers. $A$, A series of population histograms depict the spatiotemporally complex responses of a single SI cortical ensemble to three different whiskers (D2, B2, and E4). The $x$-axis represents poststimulus time (in milliseconds), the $y$-axis represents the neurons in the ensemble, and the $z$-axis represents firing rate (spikes per second). Each whisker elicits a unique spatiotemporal profile of ensemble activity. $B$, The minimal latency ( $x$-axis) and firing rate ( $y$-axis) of each neuron in the ensemble responses depicted in $A$ are plotted against each other. The location of single neurons within this two-dimensional "activity field" changes as a function of stimulus location. Note that although there are 25 neurons in $A$, there are only 24 in these plots. This is because one neuron did not respond significantly to these three whiskers.

ensemble spike trains were normalized and preprocessed using principal component analysis (PCA). PCA reduced the dimensions of the data set, a necessary step for the proper application of LDA (as opposed to LVQ) given the large number of variables and trials used in this study. The first 15 principal components calculated for each trial were used as the input matrix for this analysis. Training and testing trials were divided the same as in the LVQ ANN analyses with four-way cross-validation, and discriminant functions were derived by training on normal data and testing with "shifted" data. Ensemble performance using LDA on principal components and LVQ on raw data were statistically identical (Nicolelis et al., 1998).

In another set of experiments, the spike shifting procedure was applied to both the train and testing trials for the LDA. This prevented the LDA from building a statistical model using the normal data with temporal relations intact and then testing with spike-shifted data. Only information contained in the temporal modulation of firing rate for each neuron independently within an ensemble was available, whereas any information contained in the relationships between neurons was eliminated.

\section{Histology}

The location of each microwire was confirmed for every animal through examination of Nissl-stained sections. After completion of recording sessions, animals were deeply anesthetized with a lethal dose of pentobarbital and then perfused intracardially with $0.9 \%$ saline solution followed by $4 \%$ formalin in $0.9 \%$ saline. Brains were post-fixed for a minimum of $24 \mathrm{hr}$ in the same fixative solution. Coronal sections of the whole brain $(80 \mu \mathrm{m})$ were cut on a freezing microtome. Sections were then counterstained for Nissl. Microwire tracks and tip positions were located using a light microscope. Because microwires were chronically implanted and remained in the brain for several days, electrode tracks and tip positions could be readily identified by glial scars, obviating the need for electrical lesions.

\section{RESULTS}

Ensembles of well-isolated single neurons were recorded in the SI cortex and/or VPM thalamus from nine animals (SI cortex alone, $n=3$; VPM nucleus alone, $n=3$; SI cortex and VPM nucleus, $n=3)$. The average SI cortical ensemble size was 34 neurons (range $=26-46$ neurons), whereas the average VPM ensemble was 31 neurons (range $=26-35$ neurons). Animals with both cortical and thalamic implants were analyzed separately from those with single implants; these dual-implanted animals had an average of 38 neurons for cortical implants and 43 neurons for VPM implants.

Using our experimental approach, the average receptive field (RF) size for SI cortical neurons has been estimated to be 8.5 whiskers (Ghazanfar and Nicolelis, 1999), and the average RF size for VPM neurons is 13.7 whiskers (Nicolelis and Chapin, 1994). Stimulation of a given single whisker can elicit a spatiotemporally complex response from a large extent of both SI cortex and VPM thalamus. Figure $2 A$ depicts population histograms, which illustrate that the stimulation of different single whiskers can elicit unique large-scale distributed responses within the same ensemble of simultaneously recorded SI cortical neurons. Analyses of these responses (Fig. 2B) illustrate that the ensemble response to a single whisker stimulus was characterized by a unique distribution of individual neuron firing rate and minimal latency. Similar results have been reported for VPM ensembles (Nicolelis and Chapin, 1994). Can such spatiotemporal neural 
activity patterns be used to identify the location of a tactile stimulus on a single trial basis?

\section{Comparison of single neuron versus neural ensemble performance discriminating among 16 whiskers on a single trial basis}

Using the LVQ-based ANN, the ability of single cortical or thalamic neurons to correctly identify the location of 1 out of 16 whisker possibilities on a single trial basis was tested. This 1 out of 16 whisker discrimination set was used in many of our analyses because it circumvented the animal-to-animal variation in the placement of our microwire arrays or bundles.

Figure $3 A$ shows that single SI cortical neurons (black bars, $n=$ 60) correctly classified the tactile location on $8.68 \pm 1.9 \%$ of the trials (mean $\pm \mathrm{SD}$; range $=6.06-13.07 \%$ ), whereas single VPM neurons (gray bars, $n=63$ ) performed significantly better (unpaired $t$ test, $t_{(121)}=-2.34, p<0.05$ ), correctly classifying on average $10.85 \pm 6.95 \%$ of trials (mean $\pm \mathrm{SD}$; range $=5.69$ $39.54 \%$ ). Because chance performance for 1 out of 16 whiskers was $6.25 \%$, individual neurons performed slightly above chance.

We next investigated how much neural ensembles outperformed single neurons. Figure $3 B$ shows that SI cortical ensembles (average ensemble size $=34$ neurons) performed correctly on $46.36 \pm 3.55 \%$ (mean $\pm \mathrm{SD}$; range $=40.91-51.42 \%)$ of trials, and VPM neural ensembles (average ensemble size $=31$ neurons) performed correctly on $68.34 \pm 12.23 \%$ (mean $\pm \mathrm{SD}$; range $=50.73-81.66 \%)$ of trials. On average, SI cortical ensembles performed 7.4 times better than chance and 5.3 times better than the average single cortical neuron. Along the same lines, VPM ensembles performed 10.9 times better than chance and 6.3 times better than the average single VPM neuron. Overall, VPM ensembles performed significantly better than SI cortical ensembles (unpaired $t$ test, $t_{(22)}=-4.33, p<0.0005$ ). These results indicated that although the firing patterns of single neurons in the thalamocortical pathway can be used to identify the location of a single whisker slightly above chance levels, small neural ensembles could perform several times better than chance and several times better than even the best single neurons.

\section{Graceful degradation of ensemble performance}

We tested whether SI cortical and VPM ensembles exhibited graceful degradation in performance after the sequential removal of "best predictor neurons" (see Materials and Methods). Figure $4 A$ shows the results of this analysis for two comparably sized neural ensembles from SI cortex (top panel) and VPM (bottom panel). Both the SI cortical and VPM neural ensembles exhibited a smooth ("graceful") degradation of performance on discriminating among four different whiskers (B1, E1, B4, and E4). Similar curves were seen for all other animals (data not shown). In most cases, there was a smooth decay in ensemble performance, and chance performance was not reached until only a few neurons remained in each of the ensembles. Notice that although individual neurons were sampled from multiple barrel cortical columns (Ghazanfar and Nicolelis, 1999) or barreloids (Nicolelis and Chapin, 1994), their contribution to the discrimination of different, nonisomorphic whiskers was significant. If the neurons were only local feature detectors, then one would expect to see sharp drops in ensemble performance as the neurons dedicated to a particular whisker were removed. These results suggest that despite their anatomically modular organization, the functional organization of the thalamocortical pathway in rats is one of a highly distributed system, at least for the encoding of the location of punctate tactile stimuli by layer V cortical and VPM neurons.
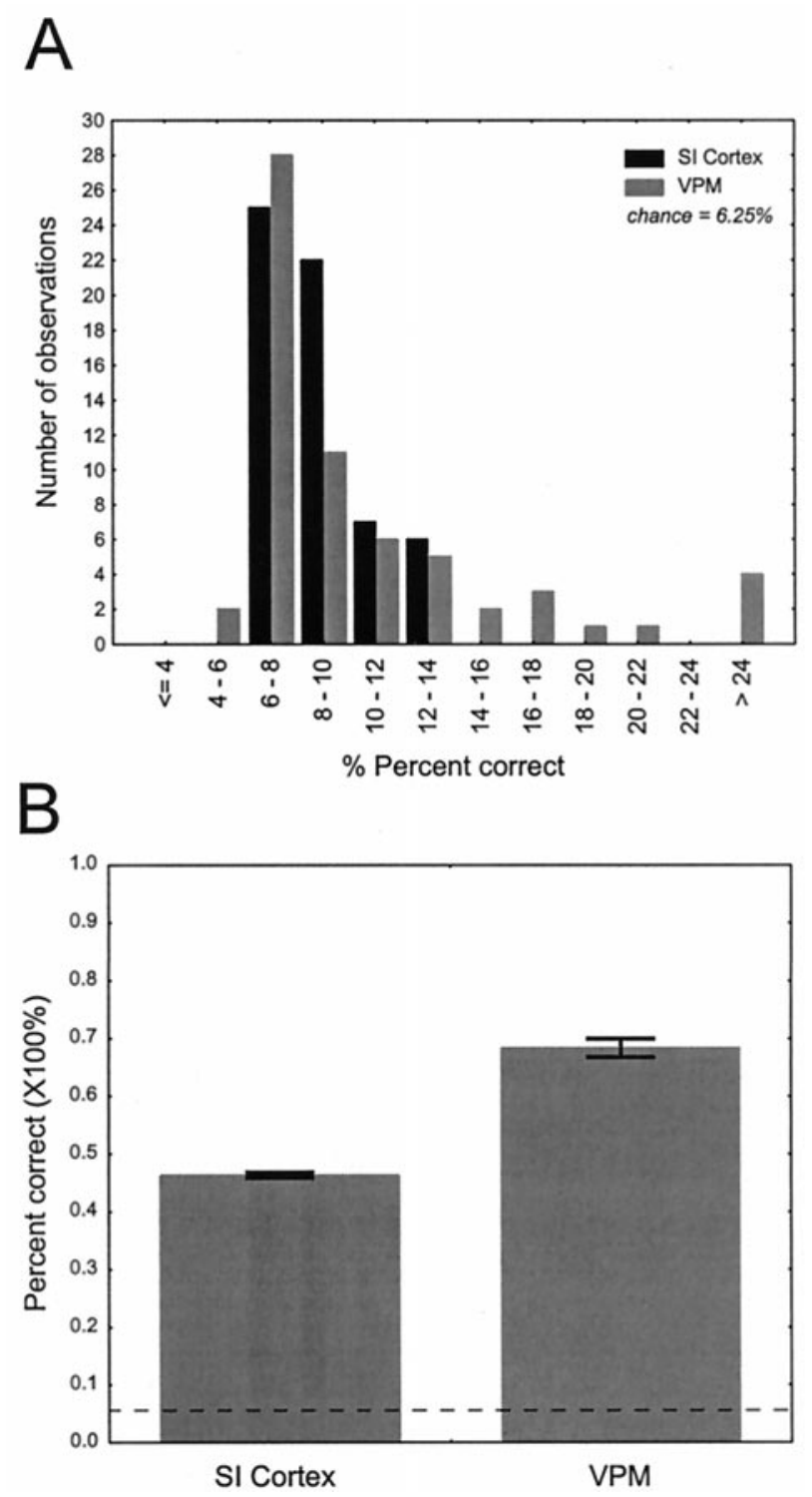

Figure 3. The discrimination capability of single neurons versus neural ensembles. $A$, Single SI cortical and VPM neurons were tested on their ability to discriminate the location of a whisker stimulus among 16 different possibilities. The $x$-axis represents the percentage of correct classified trials, and the $y$-axis represents the number of neurons. Black bars: SI cortical neurons. Gray bars: VPM neurons. Chance performance was $6.25 \%$. On average, both cortical and thalamic neurons performed slightly above chance levels. $B$, Ensembles of SI cortical and VPM neurons were similarly tested on their ability to discriminate among 16 whiskers. The percentage of correct trials is plotted on the $y$-axis. It can be seen that ensembles of neurons perform several times better than chance and several times better than the average single neuron. Furthermore, VPM ensembles perform better than SI cortical ensembles. Chance performance was $6.25 \%$, as indicated by the dashed line. Error bars show 1 SEM.

A comparison of the rate of decay in performance was made between cortical and thalamic ensembles. Because of the variable size of ensembles, this measurement was taken between $n$ and $n-10$, where $n$ equals the number of neurons in the ensemble. On average, SI cortical ensembles (2.1\% per neuron) decayed slower than VPM ensembles (3.4\% per neuron) (unpaired $t$-test, $t_{(30)}=$ 3.66, $p<0.001$ ). This suggests that a smaller number of VPM neurons carry the information for encoding tactile stimulus loca- 


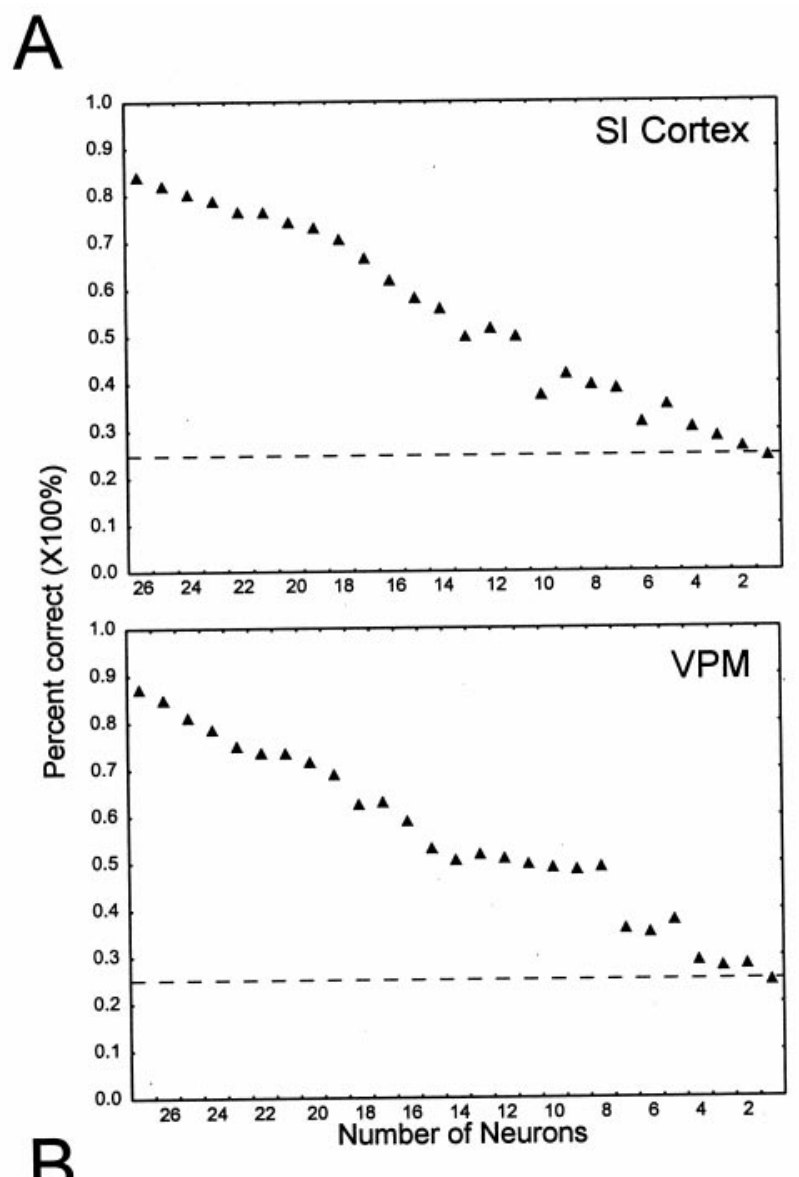

B

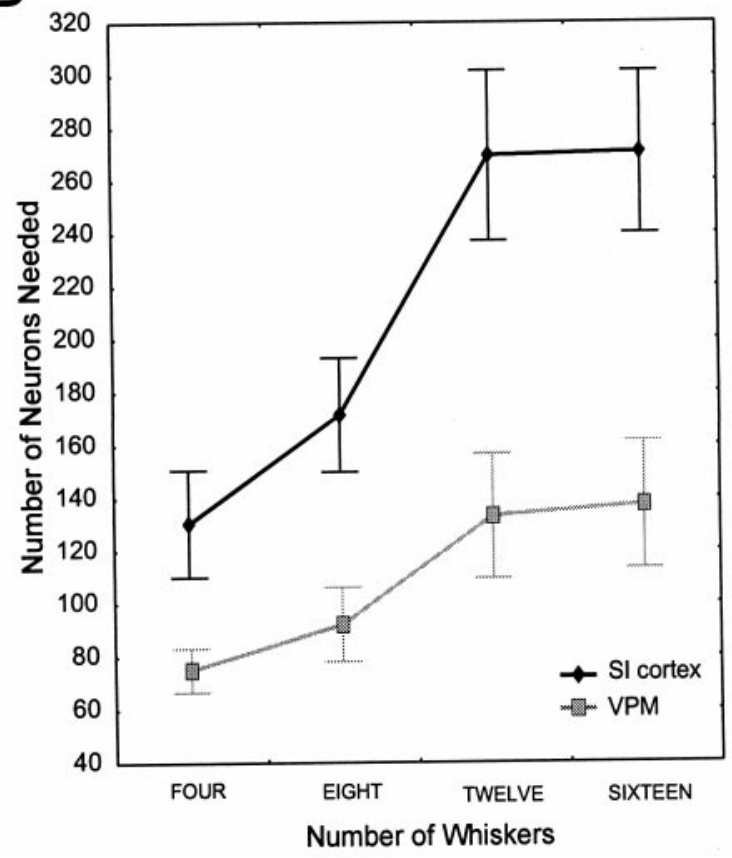

Figure 4. Distributed coding properties of cortical and thalamic ensembles. $A$, Degradation of ensemble performance discriminating four different whiskers (B1, B4, E1, and E4) was measured after the sequential, one-by-one removal of the best predictor neuron. Here it can be seen that the sequential removal of SI cortical neurons resulted in the graceful degradation of one cortical ensemble performance (top panel). All other cortical ensembles showed the same effect. Similarly, the performance of a thalamic ensemble also gracefully degraded when neurons are removed one by one (bottom panel). Chance performance was $25 \%$, as indicated by tion when compared with SI cortex. This is in accordance with both the single neuron and ensemble data from the VPM nucleus.

In an effort to estimate the minimal size of thalamic and cortical ensembles capable of discriminating the location of a whisker at a $99.9 \%$ level, a power equation was used to extrapolate from each subject's neural ensemble performance discriminating 4, 8, 12 and 16 whiskers (see Fig. 6 for whisker identities). As expected, for both SI cortex and VPM, more neurons were needed to achieve $99.9 \%$ performance on increasingly difficult discriminations (Fig. 4B). Based on the LVQ ANN, 99\% discrimination of one out of four whiskers would require on average 129 SI neurons and 75 VPM neurons. A 1 out of 16 whisker discrimination would require 269 cortical and 137 thalamic neurons. For all discrimination sets, almost twice as many cortical neurons were needed than VPM neurons. Interestingly, the number of neurons needed seemed to plateau between discriminations among 1 out of 12 and 1 out of 16 whiskers for both SI cortex and VPM neurons - the required ensemble size did not increase linearly with the complexity of the discrimination. This is another hallmark of distributed coding. Above a given ensemble size, some collective property of the neuronal population could account for this effect. Therefore, we next investigated what form such a collective property may take.

\section{Interaction between rate and temporal coding in cortical and thalamic neural ensembles}

To investigate how the temporal modulation of neural ensemble firing affected the discrimination of tactile location on a single trial basis, we parametrically increased the size of the integration window (i.e., bin size) used to generate the input vector for our LVQ ANN analysis. By increasing the bin sizes, we degraded the temporal resolution of the population signal but kept the number of spikes produced by the ensemble constant. This effect can be seen in Figure $5 A$ where the same SI cortical ensemble response to a single whisker is plotted with different bin sizes $(3,6,10$, and $20 \mathrm{msec}$ ). Notice that although temporal information was degraded by this manipulation, simple firing rate differences could conceivably be used to discriminate among different whisker deflections. Figure $5 B$ shows the performance of SI cortical and VPM ensembles when the LVQ algorithm was used to measure their ability to discriminate among 1 out of 16 whiskers using different bin sizes $(1,3,6,10,20$, and 40 msec bins). For both SI cortical and VPM ensembles, discrimination performance degraded significantly when the bin size was increased to $10 \mathrm{msec}$ (SI cortex: 6 vs $10 \mathrm{msec}, t_{(15)}=4.15, p<0.001$; VPM: 6 vs 10 msec, $\left.t_{(15)}=5.58, p<0.0005\right)$. For cortical ensembles, a bin size of $1 \mathrm{msec}$ actually degraded performance significantly (vs $3 \mathrm{msec}$ bins, $\left.t_{(11)}=-0.03, p<0.0005\right)$. Further reduction in performance was observed as bin sizes were increased beyond $10 \mathrm{msec}$. Nevertheless, both SI cortical and VPM ensembles still per-

the dashed line. $B$, The number of neurons needed within a cortical or thalamic population to achieve $99.9 \%$ correct performance was extrapolated from their average performance discriminating 4, 8, 12, and 16 whiskers. The number of cortical neurons needed to achieve near-perfect performance was approximately twice as much for discriminating 16 whiskers versus 4 whiskers (black line). Similarly, the number of neurons necessarily increased with increasing discrimination difficulty. Interestingly, for both SI cortical and VPM ensembles, the number of neurons needed reached plateau between 12 and 16 whiskers, suggesting that the number of neurons needed is not linearly related to the number of classes to be discriminated. 
A

$3 \mathrm{~ms}$ bins
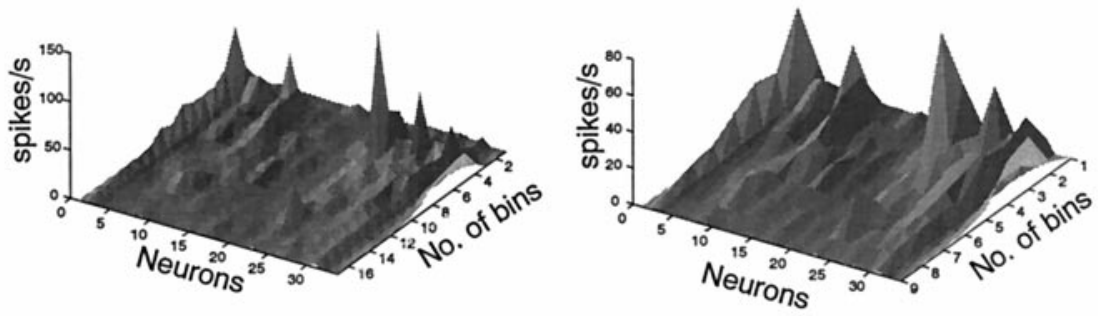

$6 \mathrm{~ms}$ bins
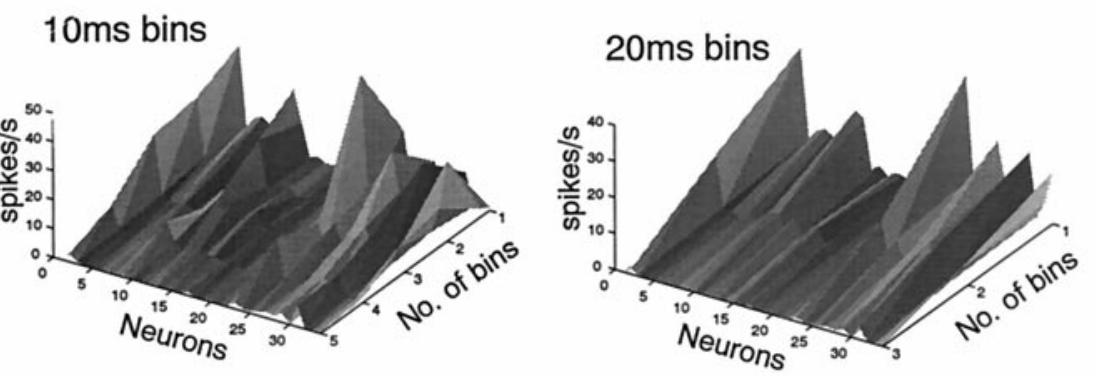

$\mathrm{B}$
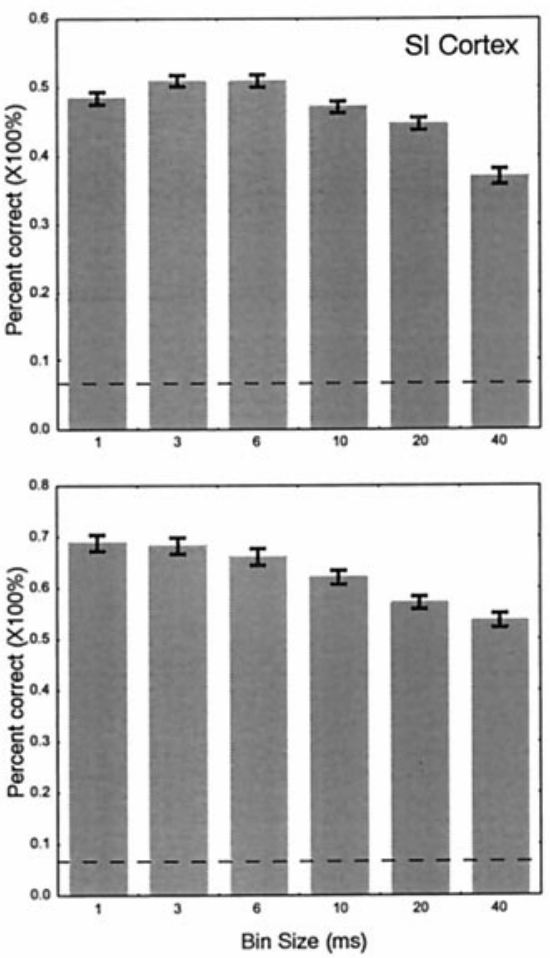

Figure 5. Interaction between rate and temporal coding. A, A series of population histograms depict the effects of increasing bin size (3, 6, 10, and 20 $\mathrm{msec}$ ) on the temporal resolution of ensemble responses. The $x$-axis represents the number of bins, the $y$-axis represents the neurons, and the $z$-axis represents the response magnitude. As shown here, such temporal manipulations preserve the overall number of spikes in the response but destroy the temporal resolution. $B$, The interaction between rate and temporal coding was tested on SI cortical and VPM thalamic ensembles discriminating among 16 whiskers. Ensemble performance was systematically tested with different bin sizes $(1,3,6,10,20$, and 40 msec). It can be seen that performance degraded significantly for both cortical and thalamic ensembles when bin sizes $>6$ msec were used. This suggests that the temporal distribution of spikes conveys important information regarding tactile stimulus location. Chance performance was $6.25 \%$, as indicated by the dashed line.

formed at greater than chance levels when only the overall average firing rate within a trial was used (40 msec bins). Thus, although the temporal modulation of ensemble response conveyed significant information about stimulus location, the total number of spikes seemed to contribute a larger proportion of information under these particular experimental conditions.

\section{Effects of disrupting correlated activity among neurons} Spike shifting and trial shuffling of testing trials

Because the activity of a large number of neurons was recorded simultaneously, we could test whether the covariance structure within ensemble responses contributed to ensemble performance. Because the LVQ algorithm is not particularly suited to analyzing the covariance structure among neurons, LDA, which explicitly looks for covariance structure, was applied to our ensemble data sets. LDA was applied before and after temporally shifting the ensemble spike trains $\pm 6-12$ msec relative to each other (spike shifting) and before and after randomly shuffling the trials of individual neuron spike trains (trial shuffling). In these data manipulations, first, a set of linear discriminant functions was derived using normal, unshifted trials. Next, three sets of new trials (which were not used to derive the discriminant functions) were used to measure the ability of these discriminant functions to predict the location of a stimulus on a single trial basis. These three sets included (1) normal, unshifted trials, (2) trials in which the timing of individual spikes for each neuron had been shifted relatively to each other (spike shifting), and (3) a set of data in which the order of trials was randomized for each neuron tested with the shifted data set (trial shuffling). The time range for spike shifting was selected based on the degradation of ensemble performance when the temporal resolution was decreased from 6 to $10 \mathrm{msec}$ (Fig. 5B). To assess the possibility that correlated activity may play different roles when different numbers of whiskers are used (i.e., increasing the difficulty of the discrimination), the same analysis was performed on thalamic and cortical ensemble responses to different combinations and numbers of whiskers (1 out of $4,8,12$, or 16 whiskers).

Spike shifting of single trial neuronal responses resulted in an overall increase in the variance calculated from mean ensemble responses $\left(0.4789\right.$ vs $\left.0.4918 ; t_{(15)}=-2.18, p<0.05\right)$. This value was obtained by measuring the change in variance of each trial's ensemble response from the mean ensemble response before and after the spike shifting procedure. As result of this increase in variance, Figure 6 shows that in every case, for both SI cortex and VPM neurons, disrupting the correlated activity between neurons using the spike shifting procedure resulted in a significant decrease in ensemble performance, regardless of which whisker combination was used. In SI cortex, for example, single trial discrimination of the location of a stimulus in one out of four whiskers dropped from $72.36 \pm 6.91 \%$ (mean \pm SD) correct to $47.17 \pm 5.72 \%$ (mean $\pm \mathrm{SD}$ ) correct after the correlated activity 


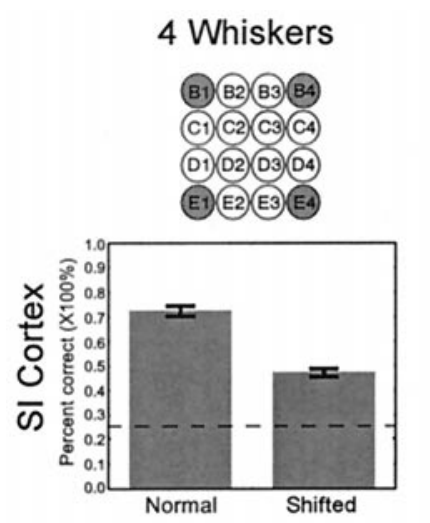

8 Whiskers
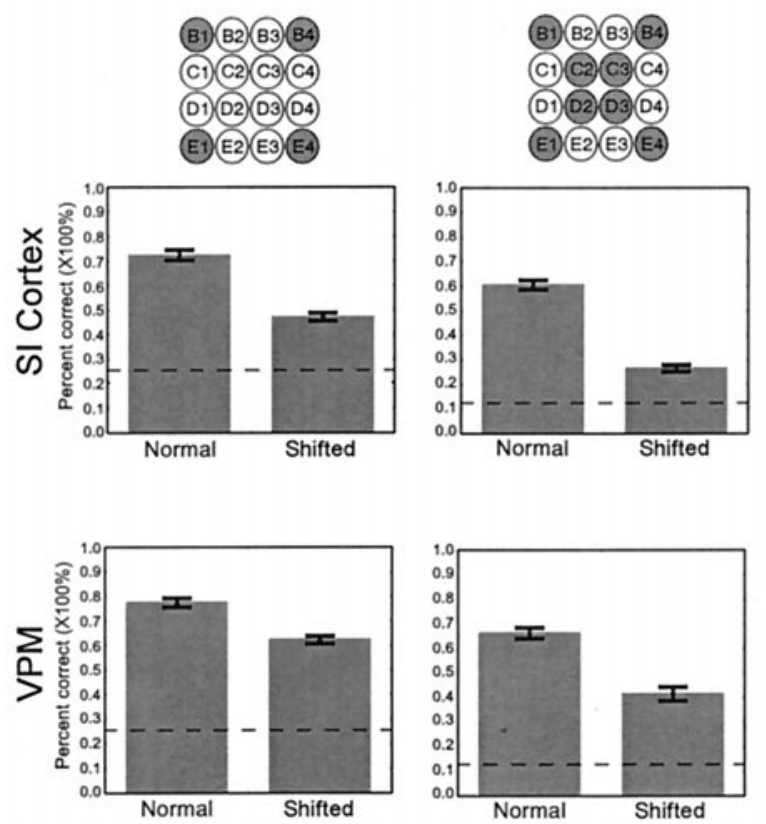

12 Whiskers
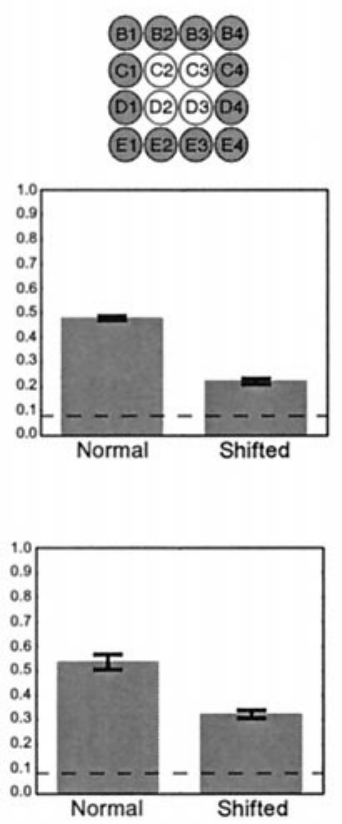

16 Whiskers
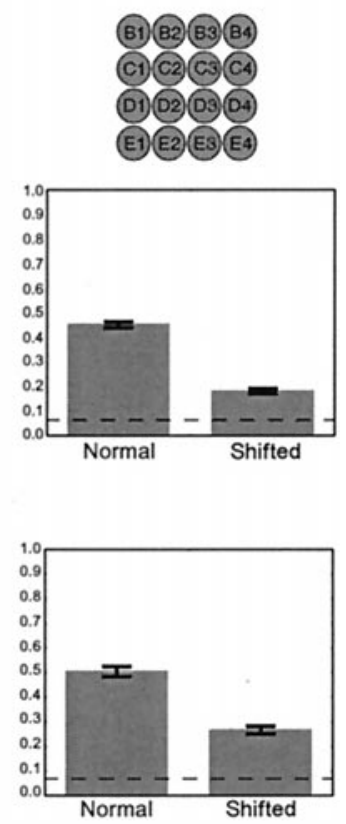

Figure 6. Effect of disrupting the correlated activity between neurons on ensemble performance. The effect of shifting spike trains relative to each other within an ensemble response was measured using linear discriminant analysis. Only spike trains of the testing trials were shifted randomly between \pm 6 and $12 \mathrm{msec}$. For both SI cortex (top panels) and VPM (bottom panels) neurons, ensemble performances were significantly worse after the covariance structure among neurons within ensembles was disrupted, regardless of the difficulty of the discrimination task $(4,8,12$, and 16 whiskers; the spatial patterns are depicted at the top of the figure). Chance performance is indicated by a dashed line in each graph.

between neurons was disrupted, a difference of $\sim 35 \%$. Similarly, for VPM neurons, correct discrimination of one out of four whiskers dropped from $77.38 \pm 6.44 \%$ (mean \pm SD) correct to $62.28 \pm 5.40 \%$ (mean $\pm \mathrm{SD}$ ) correct after correlated activity.

The magnitude of the effect of disrupting correlated activity varied according to the difficulty of the discrimination and whether the ensembles were located in SI cortex or VPM thalamus. This was measured by taking the difference between normal and spike-shifted performance and dividing this by the value obtained for normal performance [i.e., (normal - spike-shifted)/ normal]. The effect of disrupting the temporal relationships between neurons was greater when the location of the stimulus had to be identified from a larger number of whiskers for both SI cortex and thalamus (two-way ANOVA, $F_{(3,88)}=36.93, p<$ $0.000001)$. The same analysis revealed that correlated activity between neuronal firing played a larger role in cortical ensemble performance than thalamic ensemble performance $\left(F_{(1,88)}=\right.$ 17.23, $p<0.0001)$.

Next, trial shuffling was used to evaluate whether intratrialcorrelated activity across the neural ensemble played any role in coding tactile location. To measure this potential coding strategy, the spike trains of each neuron were randomly replaced with the spike train of the same neuron from another trial. Shuffling of the trial order for each neuron resulted in an overall decrease in the variance as measured by calculating the variance of each trial's ensemble response relative to the mean ensemble response before and after trial shuffling $\left(0.4789\right.$ vs $0.2455 ; t_{(15)}=10.57, p<$ 0.00001). Figure 7 shows that for both SI cortex and VPM neurons, ensemble performance discriminating among one out of four whiskers was minimally affected (ensembles performed slightly better) or not at all by trial shuffling, especially when compared with spike shifting. Similar results were obtained for 1 out of 8,12 , and 16 whisker sets (data not shown). Because shifting spike trains temporally relative to each other within a trial disrupted performance (but shuffling them across trials did not), it appears that the temporal relationships between spike trains within an ensemble response played a significant role in identifying stimulus location.

\section{Spike shifting both the training and testing trials}

By spike shifting both the training and testing trials in the LDA, we were able to prevent the analysis procedure from using any form of correlated activity to build its statistical model. In comparison with the performance of intact cortical and thalamic ensembles, we found that spike shifting both the training and testing trials of cortical and thalamic ensembles significantly disrupted performance (Fig. 8). Unlike disrupting the test trials alone, the magnitude of the effect was not different for cortical versus thalamic ensembles (two-way ANOVA, $F_{(1,88)}=0.603$, $p=0.439)$. However, as the number of whiskers increased in the discrimination set, the magnitude of the effect also increased (two-way ANOVA, $F_{(3,88)}=28.58, p<0.00001$ ). We interpret these results as suggesting that correlated activity plays a significant role in the coding of tactile location in both thalamic and cortical ensembles and that the role of correlated activity as a coding dimension increases as function of the number of whiskers to be discriminated. However, disrupting training and testing trials did not have a greater effect on coding tactile location than did disrupting testing trials alone. Across all whisker sets, disrupting testing trials alone had a greater effect on both cortical and thalamic ensembles than spike shifting training and testing trials as measured by magnitude differences in performance (SI cortex: $t_{(47)}=-7.38, p<0.00001$; VPM: $\left.t_{(47)}=-29.14, p<0.001\right)$. 


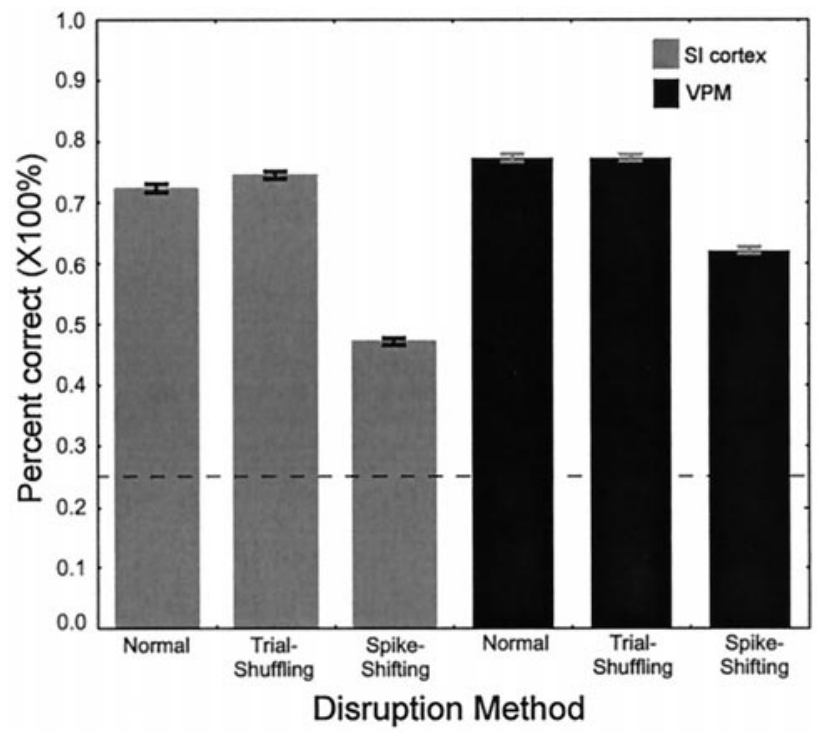

Figure 7. Influence of intratrial correlations on ensemble performance. Another method of disrupting the covariance between neurons, trial shuffling, was applied to cortical and thalamic ensembles. With this method, the spike train neurons were randomly removed and replaced with spike trains from the same neurons from other trials. This disrupted any potential intratrial covariation. As seen in this Figure, this method did not degrade ensemble performance for SI cortex (gray bars) or VPM (black bars) neurons as measured by linear discriminant analysis. Chance performance was $25 \%$, as indicated by the dashed line.

\section{Temporal evolution of ensemble performance}

Because the sensory responses of SI and VPM neurons exhibited considerable modulation over poststimulus time (Nicolelis and Chapin, 1994; Ghazanfar and Nicolelis, 1997, 1999), we investigated the poststimulus time course of SI cortical and VPM ensemble performance in discriminating among 1 out of 16 whiskers. We did this by dividing the poststimulus activity into eight $5 \mathrm{msec}$ poststimulus time epochs. Figure $9 A$ demonstrates that VPM ensemble coding was bimodal, peaking around 5-15 msec and then again at $25-30 \mathrm{msec}$, whereas SI cortical ensemble coding peaked between 10 and $20 \mathrm{msec}$. In addition, above chance performance occurred in both structures concurrently for several milliseconds. In support of this result, Figure $9 B$ depicts the simultaneously recorded activity of SI cortical and VPM ensembles after the deflection of a single whisker. After an initial activation of VPM ensembles between 5 and $15 \mathrm{msec}$, activity was concurrent between SI cortex and VPM ensembles for several milliseconds. Cortical activity peaked between 15 and $30 \mathrm{msec}$, in agreement with the ensemble performance analysis. Although not unequivocal, this temporal pattern of ensemble performance is suggestive of reverberatory activity between SI cortical and VPM ensembles and their collective involvement in the discrimination of stimulus location.

\section{Discrimination performance of simultaneously recorded thalamocortical ensembles versus SI cortex or VPM ensembles alone}

To further explore the interactions between these structures, SI cortical and VPM ensembles were recorded simultaneously in three animals, and the performance of both ensembles together was compared with the performance of each structure alone in discriminating one out of four whiskers (B1, B4, E1, and E4). As shown in Figure 10, thalamocortical ensembles performed signif-
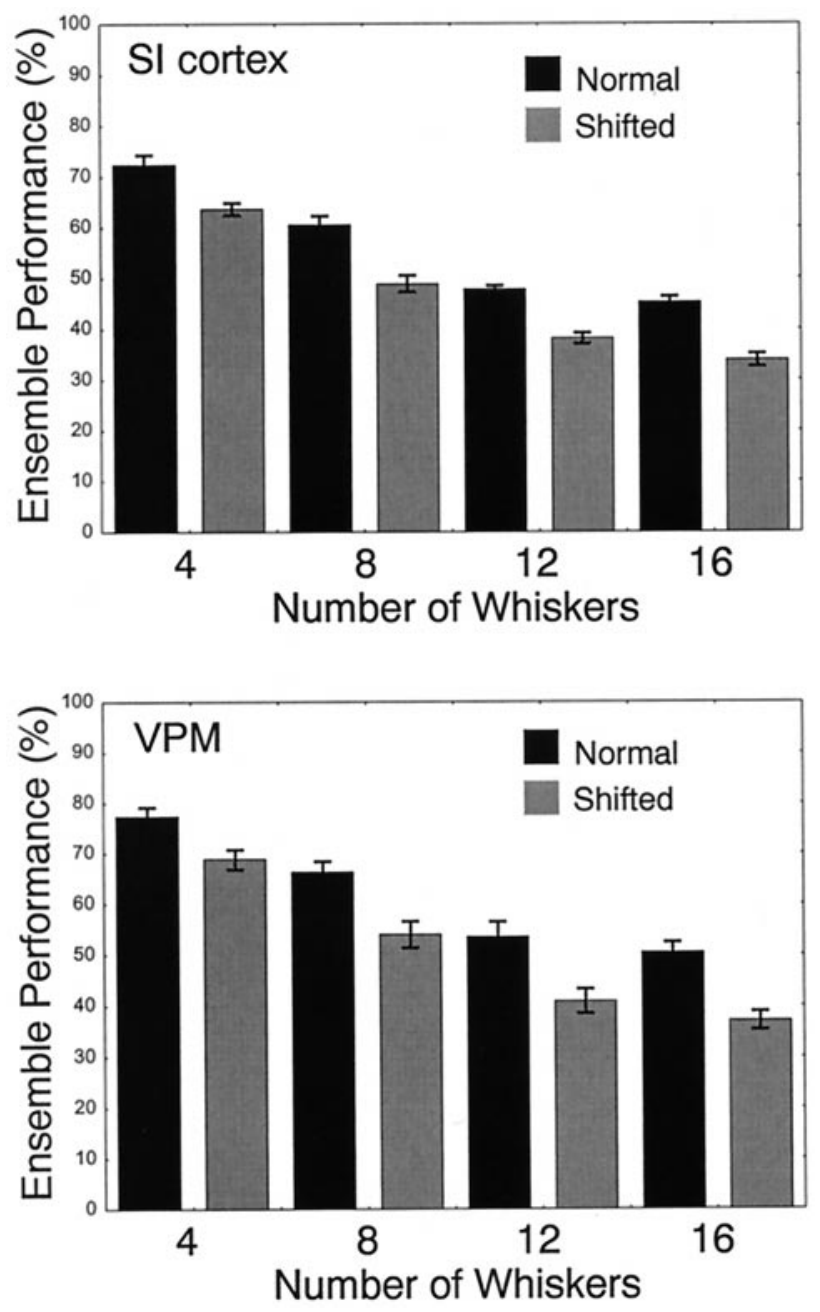

Figure 8. The effect of spike shifting on both the training and testing trials was used to measure the influence of correlated activity in ensemble coding. Decorrelating activity significantly degraded ensemble performance for both cortical and thalamic ensembles and for all sets of whiskers (see Fig. 4 for whisker identities).

icantly better than either SI cortex alone $\left(t_{(11)}=-7.50, p<\right.$ $0.00005)$ or VPM alone $\left(t_{(11)}=-7.06, p<0.0005\right)$. However, the increase in performance when SI cortex and VPM neurons were combined was not additive, suggesting that there was a degree of redundant information between these structures. Thus, simply increasing the number of neurons within a somatosensory thalamocortical ensemble did not necessarily increase performance in a linear fashion.

In support of the contention that the SI cortex and VPM neurons function interactively, Figure $11 A$ shows a plot of the effects of dropping the best predictor neuron from a thalamocortical ensemble of 75 neurons discriminating among four whiskers (B1, B4, E1, and E4), where $79 \%$ of trials were correctly classified. If the two structures were independent, one would expect to see a sharp drop in performance as the ensemble switches from dropping VPM neurons to SI cortical neurons, because VPM neurons perform, on average, better than SI cortical neurons (Fig. 3); instead, graceful degradation was still present when the two structures were combined. Figure $11 B$ depicts the graceful degradation of SI cortical and VPM ensembles when considered separately. Again, the linear 

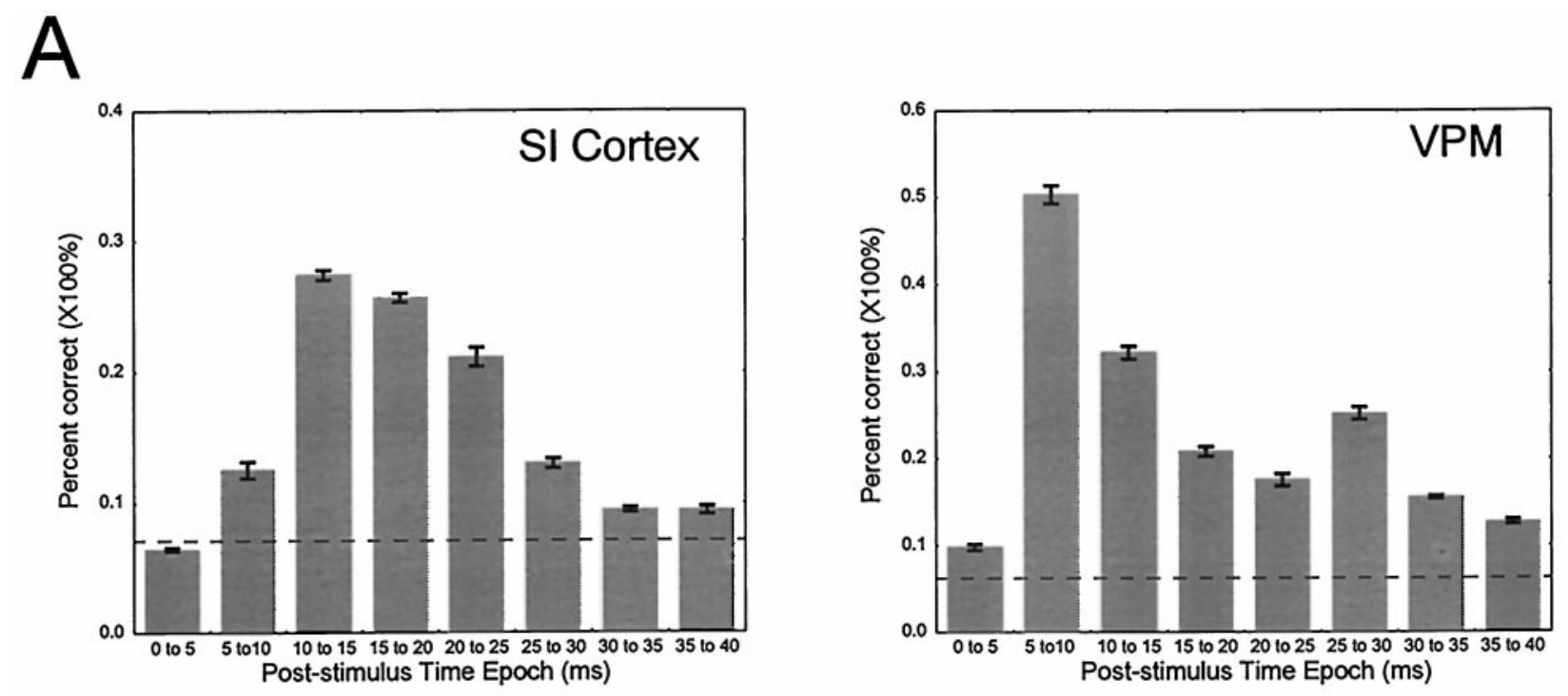

B
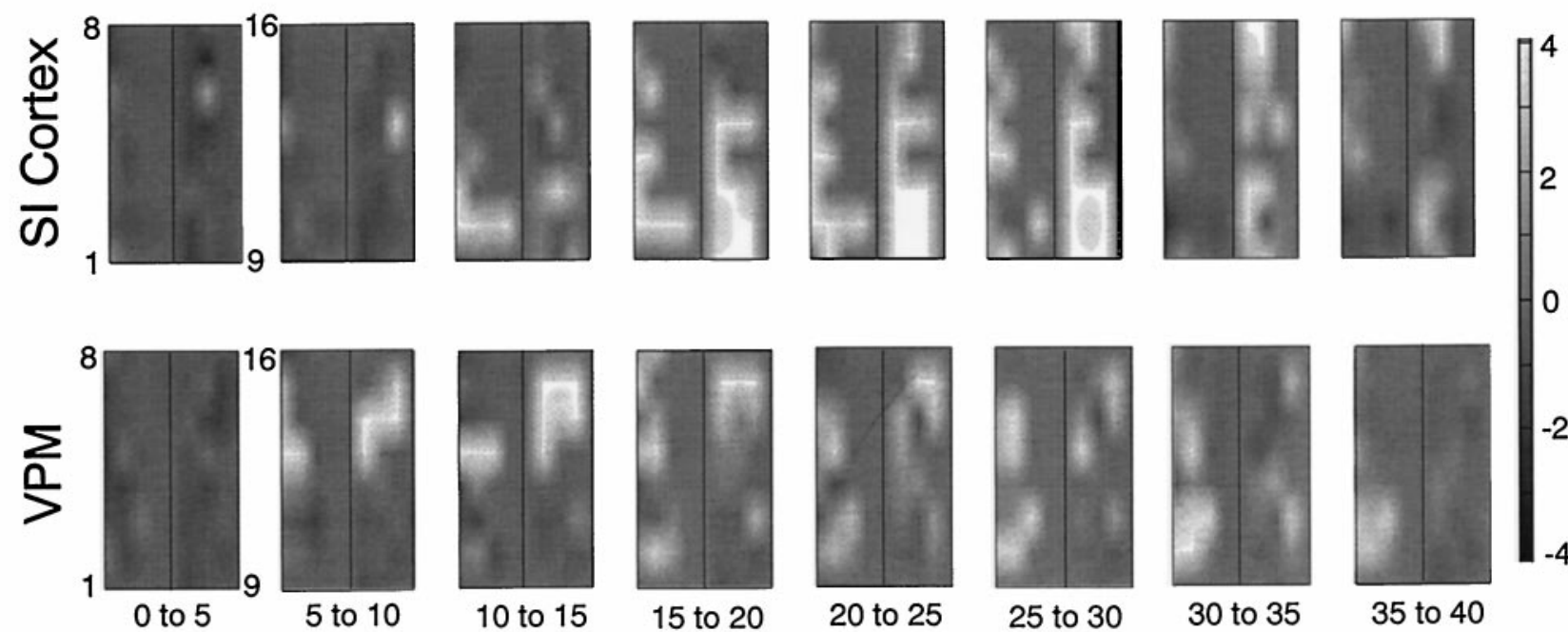

Post-stimulus time (ms)

Figure 9. Temporal evolution of ensemble performance. $A$, The time course of SI cortical (left) and VPM (right) ensemble performance discriminating among 16 whiskers was measured by dividing ensemble responses into $5 \mathrm{msec}$ poststimulus time epochs (bin size within an epoch was $1 \mathrm{msec}$ ). SI cortical ensembles peaked at 10-20 msec, whereas VPM ensembles peaked at 5-15 msec and then again at 25-30 msec. Better than chance level performance occurred concurrently for several milliseconds between these two structures. $B$, Here, raw activation plots of simultaneously recorded SI cortical and VPM ensembles demonstrate that activity between these two structures, after the deflection of a single whisker, occurred concurrently for several milliseconds poststimulus time. Three-dimensional matrices were used to represent the poststimulus firing of neurons in VPM and SI neurons according to their location on the $2 \times 8$ electrode arrays implanted in each of these structures. In each electrode array (represented by two panels plotted side by side and separated by an empty space), the $x$-axis represents the mediolateral position (left $=$ medial) of the neurons in the recording probe; the $y$-axis represents the rostrocaudal position $(t o p=$ rostral-most wires 1 and $9 ;$ bottom $=$ caudal-most wires 8 and 16$)$; and the $z$-axis, plotted in a gray-scale gradient, represents the variation in neuronal response magnitude (white = higher than 4 SDs of the spontaneous firing rate; dark gray $=$ baseline firing rate). All sensory responses were extracted from PSTHs obtained after 360 stimulation trials.

sum of their performance would result in $>100 \%$ correct classification. Extrapolation of SI cortical and VPM ensemble performance revealed that they would require 49 and 48 neurons, respectively, to achieve $79 \%$ correct independently, $35 \%$ fewer neurons than are actually needed when both structures were combined again. This further suggests the presence of redundancy in the representation of information about tactile stimulus location across SI cortex and VPM.

\section{DISCUSSION}

We found that the rat somatosensory system, despite its anatomically modular and topographic organization, could rely on a distributed coding scheme to represent the location of tactile stimuli. Within this coding scheme, both the spatial and temporal components of the ensemble activity conveyed significant information. The important temporal aspects of coding included the 


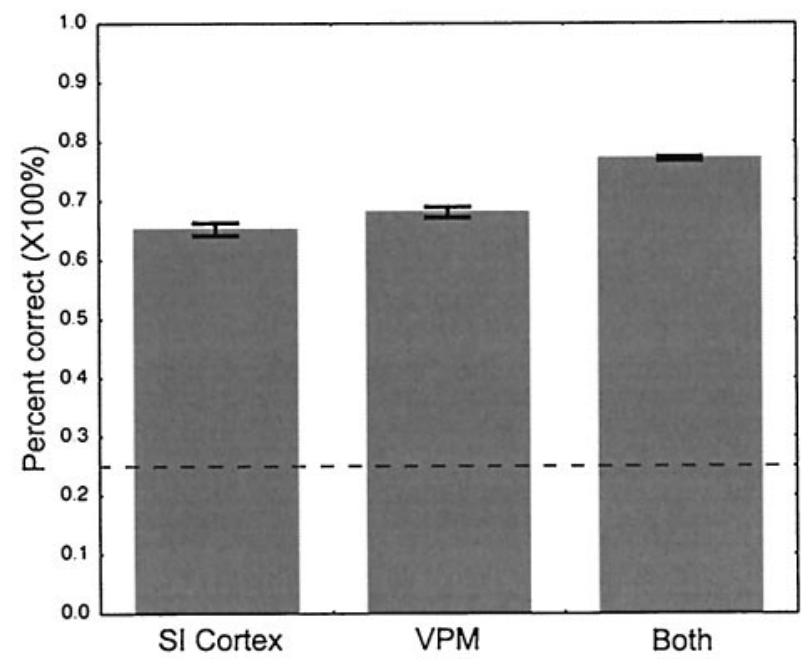

Figure 10. Redundancy of information within thalamocortical ensembles. In animals in which SI cortex and VPM ensembles were recorded simultaneously, ensemble performance discriminating among four whiskers (B1, B4, E1, and E4) was measured for each structure independently and then with the structures combined as a single ensemble. Here, combining structures did increase the performance of the ensemble but the increase was not linear, suggesting that there is a considerable amount of redundant information between SI cortex and VPM ensembles. Chance performance was $25 \%$, as indicated by the dashed line.

modulation of ensemble firing over poststimulus time and the correlated activity among neurons within ensembles. Furthermore, we obtained evidence that suggests that the relative contribution of correlated activity among neurons in coding a stimulus location changed as a function of the discrimination set. Finally, we found that the thalamus and cortex can encode tactile information concurrently and that at least some information between them is redundant, suggesting that these two structures may function as a single unit. On the basis of these results, we propose that the rat somatosensory thalamocortical pathway uses multiple strategies to encode tactile stimulus location. The extent to which a strategy is used may depend on the difficulty of the task.

Although many studies have focused on single neuron firing patterns (Richmond et al., 1990; McClurkin et al., 1991a; Middlebrooks et al., 1994, 1998; Victor and Purpura, 1996) and on pair-wise interactions between neurons (Gray et al., 1989; Ahissar et al., 1992; deCharms and Merzenich, 1996; Dan et al., 1998), there are only few studies that have examined how ensembles of single neurons function together to represent simple stimuli. To circumvent this problem, some investigators have constructed population vectors from serially recorded single units to study potential coding schemes at the level of ensembles (Georgopoulos et al., 1986; Gochin et al., 1994; Fitzpatrick et al., 1997). Despite its usefulness and the important insights gained from this approach, it does not allow one to investigate various "collective" strategies, such as temporal interactions.

The recent advent of new electrophysiological techniques that allow one to record from populations of several tens of well isolated neurons simultaneously has made such studies of ensemble coding feasible (Wilson and McNaughton, 1994; Deadwyler et al., 1996; Nicolelis et al., 1997a, 1998). Nevertheless, identifying methods for analyzing such large data sets remains a challenge (Deadwyler and Hampson, 1997). To accomplish this goal, we adopted a strategy that takes advantage of pattern recognition algorithms that use both multivariate statistical methods (Gochin
A

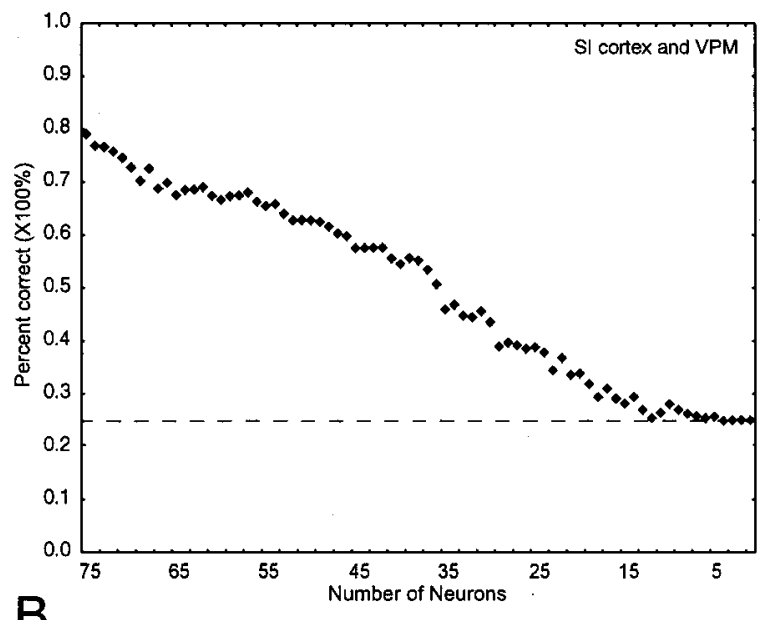

B

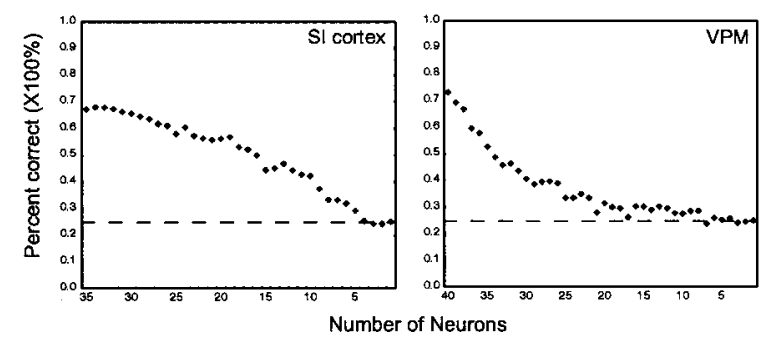

Figure 11. Graceful degradation of thalamocortical ensembles. $A$, To test further whether SI cortex and VPM ensembles function as a single entity, we combined ensembles and measured how sequentially removing the best predictor neuron affected performance discriminating four whiskers (B1, B4, E1, and E4). Depicted here is the performance of one such ensemble; it degraded gracefully. Chance performance was $25 \%$, as indicated by the dashed line. $B$, Here, the graceful degradation of the separate SI cortical and VPM ensembles is depicted. Chance performance was $25 \%$, as indicated by the dashed line.

et al., 1994; Deadwyler et al., 1996; Nicolelis et al., 1997b) and artificial neural networks (Nicolelis et al., 1998, 1999). It is important to emphasize, however, that the application of statistical pattern recognition techniques to "decode" the identity of a stimulus does not imply that these methods bear any resemblance to the actual mechanisms through which the nervous system represents tactile information. Instead, they offer us a way to quantify ways in which information could be embedded in neural activity patterns.

\section{Behavioral relevance}

Two important facts must be considered when interpreting our results. First, our data were collected from anesthetized rats. Second, our analyses were all based on whether neural ensembles can discriminate 1 out of $x$ number of whiskers. Both of these issues bear on the relevance of our data to the behaving animal. After all, during natural exploratory behaviors, rats use their whiskers actively and make multiple contacts with objects in their environment.

The dynamic and distributed nature of ensemble responses to single whisker deflections in the anesthetized rat is indistinguishable in both its temporal evolution and spatial extent from responses seen in the awake animal in certain behavioral states (Chapin and Lin, 1984; Fanselow and Nicolelis, 1999). Chapin and Lin (1984) found that SI RFs are qualitatively the same in 
both the anesthetized and awake conditions. If anything, RFs were slightly larger in the awake state. This finding is supported by more recent studies using more quantitative analyses in which it has also been shown that, depending on the behavioral state, the system appears optimized for the detection of simple stimuli (Fanselow and Nicolelis, 1999). Thus, we believe that our sensory responses in the anesthetized condition are an accurate representation of the responses one would see in at least some behavioral states in the awake animal.

Our approach in this study was to determine the extent to which ensembles could distinguish between the deflection of one whisker among others. Because the rat uses the entire caudal mystacial pad simultaneously to actively detect the spatial attributes of its environment (Carvell and Simons, 1990), we asked whether our ensembles (or single neurons) could distinguish between one whisker out of 4-16 other whiskers. Within the context of natural whisking behavior, such a task is quite reasonable. Brecht et al. (1997) have elegantly shown that the caudal whiskers, unlike the finer-grained rostral whiskers, are primarily used to detect the spatial location of objects or openings. Based on morphological and behavioral considerations, these authors conclude that the major information format in the barrel cortex is the "binary touched/untouched signal." Furthermore, it has been shown repeatedly that animals can accurately perform spatial tasks with only one whisker (Hutson and Masterton, 1986; Harris et al., 1999), showing again that a single whisker is an appropriate "sensory unit" to investigate. Within this framework, our use of the 1 out of $x$ whiskers paradigm for ensemble performance is quite reasonable. The next experimental step ought to involve more complex multi-whisker discriminations in behaving animals.

\section{Single neurons versus neural ensembles}

Our study indicated that ensembles of SI cortical and VPM neurons were several times better than single neurons at identifying the location of a stimulus on the whisker pad on a single trial basis. Moreover, ensemble performance degraded gracefully when neurons were removed, one by one, from the population. These results, combined with the fact that single whiskers can elicit a spatiotemporally complex response from a large portion of SI cortex (Armstrong-James et al., 1992; Kleinfeld and Delaney, 1996; Masino and Frostig, 1996; Moore and Nelson, 1998; Peterson et al., 1998; Ghazanfar and Nicolelis, 1999) and VPM (Nicolelis and Chapin, 1994), strongly suggest that despite their modular anatomy, the rat SI cortex and VPM neurons may rely on distributed encoding strategies to identify stimulus location on a single trial basis.

Along similar lines, in the auditory cortex of the cat, Middlebrooks et al. $(1994,1998)$ found that single neurons were also broadly tuned for sound location and suggested that a distributed population code would be required to encode the location of auditory stimuli at the accuracy levels observed psychophysically. Thus, because the activity of a broadly tuned neuron is unable by itself to provide unequivocal information concerning stimulus location, such activity is only meaningful in the context of the activity of other neurons (Erickson, 1968). Importantly, there is not a topographic map of auditory space in the cortical area where these neurons were sampled from, suggesting that such maps are not necessary for coding spatial location with great precision.

Our data suggest that single neurons or even small, localized groups of neurons are, by themselves, inefficient processors of sensory information. It has been argued, however, that individual neurons may be the dominant coding units for near-threshold stimuli. This "lower envelope principle" states that sensory thresholds are set by the sensory neurons that have the lowest threshold for the stimulus used (Barlow, 1995). Several points argue against the possibility of single neuron decoders in the rat somatosensory system. First, receptive fields mapped with smaller whisker deflections (i.e., low-threshold stimuli) than used in this study and previous studies (Nicolelis and Chapin, 1994) have comparably sized receptive fields (Armstrong-James and Fox, 1987; Simons and Carvell, 1989). Thus, the spatial tuning of neurons is similar even for smaller whisker deflections than those used in the present study. Second, an optical imaging study, in which whisker deflection amplitudes were parametrically manipulated, demonstrated that even weak stimuli elicit a response that extends well beyond a single barrel cortical column in SI cortex (Peterson et al., 1998). To reiterate, despite the anatomical modularity and topography in both SI and VPM neurons, stimulation of each whisker activates cells in locations beyond its isomorphic barrel or barreloid. Within a barrel column, for example, individual neurons receive synaptic inputs from multiple whiskers (Moore and Nelson, 1998; Zhu and Connors, 1999). Such interconnectivity necessarily gives rise to large, distributed responses.

For the barrel cortex, layer $\mathrm{V}$ neurons have, on average, the largest RFs (Simons, 1978; Chapin, 1986). It is therefore possible that single neurons or smaller groups of neurons in other barrel cortical layers could accurately distinguish the location of tactile stimuli. For example, although layer IV neurons have large and dynamic multi-whisker RFs at the subthreshold level (Moore and Nelson, 1998), these are ultimately reduced in size by inhibitory interactions resulting in suprathreshold RFs of only one or two whiskers. Nevertheless, the majority of supragranular and infragranular layer neurons of barrel cortex and VPM neurons have large, multi-whisker RFs (Simons, 1978; Chapin, 1986; Nicolelis and Chapin, 1994), and it is the infragranular neurons that are the primary source of SI output. Indeed, neurons in layer V send axons to various intracortical and subcortical targets (Killackey et al., 1989; Koralek et al., 1990). Thus, the study of layer V ensembles gives a more accurate perspective of the neural activity that downstream targets have to decode.

\section{VPM neural ensembles perform better than SI cortex}

Both single neurons and ensembles of the VPM nucleus performed better than SI cortex for 1 out of 16 whisker discriminations. This is interesting in light of the fact that VPM neurons have larger receptive fields than SI cortex; i.e., the tuning of neurons becomes sharper from thalamus to cortex (Simons, 1978; Armstrong-James and Fox, 1987; Simons and Carvell, 1989; Nicolelis and Chapin, 1994; Ghazanfar and Nicolelis, 1999). Rate of decay data also suggested that fewer VPM neurons are needed to encode stimulus location when compared with SI layer V neurons. Perhaps this difference in performance can be attributed to the fact that VPM neurons fire at a higher rate under anesthetized (Simons and Carvell, 1989; Nicolelis and Chapin, 1994; Ghazanfar and Nicolelis, 1999) and awake (Nicolelis et al., 1995) conditions and exhibit greater temporal modulation of their responses than SI cortical neurons (Fig. 9A). Thus, under these conditions VPM ensembles provide information in more dimensions for encoding stimulus attributes. In the auditory system, it was found that sharper tuning of neurons in later stages of the pathway resulted in more efficient population vector coding of sound localization than earlier stages with more broadly tuned neurons (Fitzpatrick et al., 1997). It is conceivable that this difference between our results and those of Fitzpatrick et al. (1997) 
for the auditory system is attributable to the fact that our ensemble analysis incorporated temporal information across neurons.

Although we did not test the role of this property in the current study, "bursting" may also play a role in coding stimulus location. Bursts are characterized as a series of spikes with short interspike intervals, and neurons in both layer V (Amitai and Connors, 1995) and thalamus (Godwin et al., 1996) may display bursting behavior. The relevance of bursts for information encoding and transmission in the somatosensory thalamocortical pathway awaits further study.

\section{Inseparability of rate and temporal coding parameters}

Several single neuron studies have demonstrated that the temporal modulation of firing rate can carry a significant amount of stimulus-related information (Richmond et al., 1990; McClurkin et al., 1991a; Middlebrooks et al., 1994, 1998; Victor and Purpura, 1996). In these studies, single neurons were shown to encode various features (color, spatial frequency, etc.) simultaneously when time was used as a coding dimension. These results support the idea that different features of a stimulus do not need to be encoded by distinct populations of neurons each devoted to a particular stimulus. Instead, the same population of neurons could encode multiple stimulus attributes simultaneously by using distinct encoding strategies (e.g., firing rate, time-modulation of rate, correlated activity, etc.) to represent each of these features (Nicolelis et al., 1998).

The importance of the temporal dimension in our data was demonstrated by showing that decreasing the temporal resolution of neural ensemble response resulted in significant decreases in performance for both SI cortex and VPM ensembles. Our data suggest, therefore, that both the number of spikes and the temporal modulation of the ensemble firing can carry information regarding stimulus location. Firing rate differences among neurons in SI cortex and VPM ensembles, however, were still sufficient to encode stimulus location several times above chance levels. Similar results have been reported for area SII in primates (Nicolelis et al., 1998). Thus, the ensemble coding of tactile stimulus location seems to be best represented when both the temporal modulation of the neural ensemble response and the average firing rate are taken into account.

Other forms of temporal coding - the phase relationships and potentially other forms of correlated activity-were tested by spike shifting, a procedure that randomly jitters individual spike trains relative to one another, and by trial shuffling, a procedure aimed at disrupting the intratrial covariance structure of ensemble responses. Spike shifting, but not trial shuffling, resulted in significantly degraded ensemble performance for both SI cortex and VPM ensembles. This suggests that phase relationships between the stimulus-locked modulation of firing rate changes across the ensemble encode stimulus location in the thalamocortical loop. This type of covariance structure among neurons is maintained in trial shuffling but disrupted in spike shifting.

This study also revealed that the role of correlated activity between neurons in ensemble performance increased as a function of the difficulty of the discrimination: as the number of whiskers to discriminate among increased, so did the contribution of correlated activity as a coding domain. Related to this, the number of neurons needed to encode stimulus location did not increase linearly as the number of whiskers increased but instead reached plateau between 12 and 16 whiskers (Fig. 4B). We speculate that these results may be interpreted as an indication that more neurons are not necessarily needed because of a cor- responding shift toward the increased use of different coding dimensions. This gives rise to the hypothesis that the encoding mechanism selected by the neural ensemble may be task dependent. Thus, under different circumstances, such as behavioral states (Fanselow and Nicolelis, 1999), the same neural ensemble may take advantage of distinct strategies according to the context in which a particular computation is performed.

It has been argued that covariation of neural activity and the temporal discharge patterns of cortical neurons transmit little or no information and that rapid changes in firing rate are the sole information channel for coding (Shadlen and Newsome, 1998). Our data and previous studies (Richmond et al., 1990; McClurkin et al., 1991a; Middlebrooks et al., 1994, 1998; Victor and Purpura, 1996; Dan et al., 1998; Nicolelis et al., 1998) argue for a more balanced account of the role of time in neural coding in the thalamocortical loop. Indeed, our results demonstrate that decreasing the temporal resolution of the ensemble response (but keeping spike count information unchanged) and disrupting the covariance structure among cortical and thalamic neurons can significantly degrade the discrimination performance of thalamocortical ensembles. Nevertheless, ensemble performance subsequent to both of these manipulations always remained above chance, suggesting that firing rate changes do play a major role in the transmission of sensory information.

\section{The primary somatosensory cortex and thalamus function as a single unit}

Several findings in this study argue in favor of the view that in the rat somatosensory system, SI cortex and VPM neurons function as a single entity in the discrimination of stimulus location on a single trial basis. First, measurements of the raw ensemble responses of simultaneously recorded cortical and thalamic ensembles revealed concurrent activity for several milliseconds of poststimulus time (Ghazanfar and Nicolelis, 1997). Second, the temporal analysis of SI cortical and VPM ensemble performance revealed that above chance performance occurred in both structures concurrently. Third, dropping neurons one at a time from thalamocortical ensembles resulted in the graceful degradation of performance. Finally, the two structures showed some redundancy of information in the coding of stimulus location. Coupled with the extensive data on the reciprocal anatomical connections between these two structures (Chmielowska et al., 1989; Bourassa et al., 1995), these results suggest the existence of tightly related functional neural ensembles that could be used, among other things, to encode tactile stimulus location. In support of this contention, neurons in the primate somatosensory and visual system also seem to encode information concurrently in reciprocally connected structures (McClurkin et al., 1991b; Nicolelis et al., 1998).

\section{Conclusions}

One of the potential benefits of topographic maps in the sensory systems of vertebrates is the ability to easily identify the location of a stimulus: localized groups of neurons respond specifically to the presence of stimulus in a restricted portion of the sensory space. Yet despite the precise topographic arrangement of modules along the rat trigeminal somatosensory pathway, this sensory system does not appear to restrict its encoding repertoire to a local coding scheme. Instead, our data demonstrate that a distributed coding scheme, in which the participation of a large number of neurons located in many different modules across the thalamocortical pathway is necessary, may be used by this system to compute the location of a tactile stimulus on a single trial basis. 
Within this scheme, both the spatial and temporal characteristics of neural ensemble firing convey information. Moreover, our data suggest that the strategies that the system may use change as a function of the number of stimulus locations to discriminate, a measure of the degree of difficulty.

In summary, the representation of sensory features appears to arise from the dynamic interactions among neurons within and between brain structures, which include various coding strategies. We also propose that, depending on behavioral states and/or the task at hand (or whiskers, in this context), the CNS may rely on different strategies to solve the same problem. In this framework, pure firing rate coding and multiple time codes may coexist in the same ensemble.

\section{APPENDIX}

The LVQ classification system used for this study is a multilayer, feedforward ANN with full connectivity. The first layer is simply the input or pattern vector. The second layer contains an ANU for each pattern to be discriminated. Each ANU has a weight vector with an equivalent number of elements as the input (pattern) vector. There is no bias value for these ANUs. The output value of each ANU is determined by the following Euclidean distance function:

$$
a_{\mathrm{i}}\left(\mathbf{W}_{\mathrm{i}}, \mathbf{I}\right)=-\left\|\mathbf{I}-\mathbf{W}_{\mathrm{i}}\right\|,
$$

where $a_{\mathrm{i}}$ is the output value for the $\mathrm{i}^{\text {th }} \mathrm{ANU}, \mathbf{W}_{\mathrm{i}}$ is the weight vector, and $\mathbf{I}$ is the input vector for the system. The unit with the greatest output value has the weight vector that is closest in Euclidean distance to the input vector. This is known as the "winning" ANU.

\section{The LVQ learning rule}

Each ANU is responsible for recognizing input patterns. Because the class of patterns that each of these ANUs must find is predetermined (supervised learning), it must be penalized for finding a pattern of the wrong class and rewarded for finding a pattern of the correct class. This is realized by the optimized LVQ (Kohonen 1997).

Let $c$ define the index of the winner in the second layer:

$$
c=\underset{i}{\arg \min }\left\{\left\|\mathbf{I}-\mathbf{W}_{\mathrm{i}}\right\|\right\},
$$

and let $\alpha_{\mathrm{i}}(t)$ the learning-rate factor assigned to each $\mathbf{W}_{\mathrm{i}}$, then:

$$
\mathbf{W}_{\mathrm{c}}(t+1)=\left[1-s(t) \alpha_{\mathrm{c}}(t)\right] \mathbf{W}_{\mathrm{c}}(t)+s(t) \alpha_{\mathrm{c}}(t) \mathbf{I}(t),
$$

where

$$
\begin{gathered}
s(t)=+1 \text { if } \mathbf{I} \text { is classified correctly } \\
s(t)=-1 \text { if } \mathbf{I} \text { is classified incorrectly, }
\end{gathered}
$$

and after each learning step:

$$
\alpha_{\mathrm{c}}(t)=\left[1-s(t) \alpha_{\mathrm{c}}(t)\right] \alpha_{\mathrm{c}}(t-1) .
$$

This classification system searches for patterns closest in Euclidean distance to one of our input weight vectors, so each of these weight vectors is a codebook vector that the system will use for classification. The LVQ algorithm shown moves these codebook vectors closer to properly classified input training vectors and away from improperly classified training vectors by the learning rate $\alpha_{\mathrm{i}}(t)$, which decreases after each learning step.

\section{Initialization}

The weight vectors of the ANUs are all initialized to the same value: the midpoint of the range of values of the input vectors. They differ, however, in what class of pattern they are assigned to recognize. Because this is a supervised learning algorithm, each ANU is preassigned to one class during initialization. During training, these ANUs learn to recognize the class to which they are preassigned. In general, there must be at least as many ANUs as there are classes to be discriminated, although it is possible to have more ANUs than classes. In this study, two ANUs are assigned to each class so that there are twice as many ANUs as there are classes.

\section{Training}

Training the LVQ network involves executing the LVQ learning rule for all the input vectors repeatedly until the ANU weight vectors have moved as close as possible to their assigned class of input patterns. In this study we chose a sufficiently large number of iterations to ensure that this occurs: two times the product of the number of input vector patterns and the number of ANUs. This number of training iterations was determined empirically to be sufficient such that the weights of the ANUs have become the optimal codebook vectors for classification of input patterns.

\section{Testing}

To test the LVQ network we present it with trials that were not used during training. One ANU weight vector will be the "winning" ANU, and the class to which it was preassigned is the predicted classification of the input pattern. If this matches the actual class of the input pattern, proper classification has been achieved, and if it does not then an improper classification has occurred.

\section{REFERENCES}

Abeles M, Goldstein M (1977) Multispike train analysis. IEEE Proc 65:762-773.

Ahissar M, Ahissar E, Bergman H, Vaadia E (1992) Encoding of soundsource location and movement: activity of single neurons and interactions between adjacent neurons in the monkey auditory cortex. J Neurophysiol 67:203-215.

Amitai Y, Connors BW (1995) Intrinsic physiology and morphology of single neurons in neocortex. In: Cerebral cortex, Vol 11 (Jones EG, Diamond IT, eds), pp 299-331. New York: Plenum.

Armstrong-James M, Fox K (1987) Spatiotemporal convergence and divergence in the rat SI "barrel" cortex. J Comp Neurol 263:265-281.

Armstrong-James M, Fox K, Das-Gupta A (1992) Flow of excitation within rat barrel cortex on striking a single vibrissa. J Neurophysiol 68:1345-1358.

Barlow H (1995) The neuron doctrine in perception. In: The cognitive neurosciences (Gazzaniga MS, ed), pp 415-435. Cambridge, MA: MIT.

Bourassa J, Pinault D, Deschenes M (1995) Corticothalamic projections from the cortical barrel field to the somatosensory thalamus in rats: a single-fibre study using biocytin as an anterograde tracer. Eur J Neurosci 7:19-30.

Brecht M, Preilowski B, Merzenich MM (1997) Functional architecture of the mystacial vibrissae. Behav Brain Res 84:81-97.

Carpenter AF, Georgopolous AP, Pellizzer G (1999) Motor cortical encoding of serial order in a context-recall task. Science 283:1752-1757.

Carvell GE, Simons DJ (1990) Biometric analyses of vibrissal tactile discrimination in the rat. J Neurosci 10:2638-2648.

Chapin JK, Lin CS (1984) Mapping the body representation in the SI cortex of anesthetized and awake rats. J Comp Neurol 229:199-213.

Chapin JK (1986) Laminar differences in sizes, shapes, and response profiles of cutaneous receptive fields in the rat SI cortex. Exp Brain Res 62:549-559.

Chmielowska J, Carvell GE, Simons DJ (1989) Spatial organization of thalamocortical and corticothalamic projection systems in the rat SmI barrel cortex. J Comp Neurol 285:325-338.

Dan Y, Alonso JM, Usrey WM, Reid RC (1998) Coding of visual 
information by precisely correlated spikes in the lateral geniculate nucleus. Nat Neurosci 1:501-507.

Deadwyler SA, Hampson RE (1997) The significance of neural ensemble codes during behavior and cognition. Annu Rev Neurosci 20:217-244.

Deadwyler SA, Bunn T, Hampson RE (1996) Hippocampal ensemble activity during spatial delayed-nonmatch to sample performance in rats. J Neurosci 16:354-372.

deCharms RC, Merzenich MM (1996) Primary cortical representation of sounds by the coordination of action-potential timing. Nature 381:610-613.

Erickson RP (1968) Stimulus encoding in topographic and nontopographic afferent modalities: on the significance of the activity of individual sensory neurons. Psychol Rev 75:447-465.

Fanselow EE, Nicolelis MAL (1999) Behavioral modulation of tactile responses in the rat somatosensory system. J Neurosci 19:7603-7613.

Fitzpatrick DC, Batra R, Stanford TR, Kuwada S (1997) A neuronal population code for sound localization. Nature 388:871-874.

Georgopoulos AP, Schwartz AB, Kettner RE (1986) Neuronal population coding of movement direction. Science 233:1416-1419.

Ghazanfar AA, Nicolelis MAL (1997) Nonlinear processing of tactile information in the thalamocortical loop. J Neurophysiol 78:506-510.

Ghazanfar AA, Nicolelis MAL (1999) Spatiotemporal properties of layer $\mathrm{V}$ neurons of the rat primary somatosensory cortex. Cereb Cortex 9:348-361.

Gochin PM, Colombo M, Dorfman GA, Gerstein GL, Gross CG (1994) Neural ensemble coding in inferior temporal cortex. J Neurophysiol 71:2325-2337.

Godwin DW, Vaughan JW, Sherman SM (1996) Metabotropic glutamate receptors switch visual response mode of lateral geniculate nucleus cells from burst to tonic. J Neurophysiol 76:1800-1816.

Gray CM, Konig P, Engel AK, Singer W (1989) Oscillatory responses in cat visual cortex exhibit inter-columnar synchronization which reflects global stimulus properties. Nature 338:334-337.

Harris JA, Petersen RS, Diamond ME (1999) Distribution of tactile learning and its neural basis. Proc Natl Acad Sci USA 96:7587-7591.

Hutson KA, Masterton RB (1986) The sensory contribution of a single vibrissa's cortical barrel. J Neurophysiol 56:1196-1223.

Killackey HP (1973) Anatomical evidence for cortical subdivisions based on vertically discrete thalamic projections from the ventral posterior nucleus to cortical barrels in the rat. Brain Res 51:326-331.

Killackey HP, Koralek K-A, Chiaia NL, Rhoades RW (1989) Laminar and areal differences in the origin of the subcortical projection neurons of the rat somatosensory cortex. J Comp Neurol 282:428-445.

Kleinfeld D, Delaney KR (1996) Distributed representation of vibrissa movement in the upper layers of somatosensory cortex revealed with voltage-sensitive dyes. J Comp Neurol 376:89-108.

Kohonen T (1997) Self-organizing maps. New York: Springer.

Koralek K-A, Olavarria J, Killackey HP (1990) Areal and laminar organization of corticocortical projections in the rat somatosensory cortex. J Comp Neurol 299:133-150.

Ma PM (1991) The barrelettes: architectonic vibrissal representation in the brainstem trigeminal complex of the mouse. I. Normal structural organization. J Comp Neurol 303:286-299.

MacPherson JM, Aldridge JW (1979) A quantitative method of computer analysis of spike train data collected from behaving animals. Brain Res 175:183-187.

Masino SA, Frostig RD (1996) Quantitative long-term imaging of the functional representation of a whisker in rat barrel cortex. Proc Natl Acad Sci USA 93:4942-4947.

McClurkin JW, Gawne TJ, Optican LM, Richmond BJ (1991a) Lateral geniculate neurons in behaving primates. II. Encoding of visual information in the temporal shape of the response. J Neurophysiol 66:794-808.

McClurkin JW, Optican LM, Richmond BJ, Gawne TJ (1991b) Concurrent processing and complexity of temporally encoded neuronal messages in visual perception. Science 253:675-677.

Middlebrooks JC, Clock AE, Xu L, Green DM (1994) A panoramic code for sound location by cortical neurons. Science 264:842-844.

Middlebrooks JC, Xu L, Eddins AC, Green DM (1998) Codes for soundsource location in nontonotopic auditory cortex. J Neurophysiol 80:863-881.
Moore CI, Nelson SB (1998) Spatio-temporal subthreshold receptive fields in the vibrissa representation of rat primary somatosensory cortex. J Neurophysiol 80:2882-2892.

Nelson ME, Bower JM (1990) Brain maps and parallel computers. Trends Neurosci 13:403-408.

Nicolelis MAL (1997) Dynamic and distributed somatosensory representations as the substrate for cortical and subcortical plasticity. Semin Neurosci 9:24-33.

Nicolelis MAL (1999) Methods in neural ensemble recordings. New York: CRC.

Nicolelis MAL, Chapin JK (1994) Spatiotemporal structure of somatosensory responses of many-neuron ensembles in the rat ventral posterior medial nucleus of the thalamus. J Neurosci 14:3511-3532.

Nicolelis MAL, Baccala LA, Lin RCS, Chapin JK (1995) Sensorimotor encoding by synchronous neural ensemble activity at multiple levels of the somatosensory system. Science 268:1353-1358.

Nicolelis MAL, Ghazanfar AA, Faggin BM, Votaw S, Oliveira LMO (1997a) Reconstructing the engram: simultaneous, multisite, many single neuron recordings. Neuron 18:529-537.

Nicolelis MAL, Lin RCS, Chapin JK (1997b) Neonatal whisker removal reduces the discrimination of tactile stimuli by thalamic ensembles in adult rats. J Neurophysiol 78:1691-1706.

Nicolelis MAL, Ghazanfar AA, Stambaugh CR, Oliveira LMO, Laubach M, Chapin JK, Lin RCS, Nelson RJ, Kaas JH (1998) Simultaneous encoding of tactile information by three primate cortical areas. Nat Neurosci 1:621-630.

Nicolelis MAL, Stambaugh CR, Brisben A, Laubach M (1999) Methods for simultaneous multisite neural ensemble recordings in behaving primates. In: Methods in neural ensemble recordings (Nicolelis MAL, ed). New York: CRC.

Peterson BE, Goldreich D, Merzenich MM (1998) Optical imaging and electrophysiology of rat barrel cortex. I. Responses to small singlevibrissa deflections. Cereb Cortex 8:173-183.

Richmond BJ, Optican LM (1987) Temporal encoding of twodimensional patterns by single units in primate inferior temporal cortex. II. Quantification of response waveform. J Neurophysiol 57:147-161.

Richmond BJ, Optican LM, Spitzer H (1990) Temporal encoding of two-dimensional patterns by single units in primate primary visual cortex. I. Stimulus-response relations. J Neurophysiol 64:351-369.

Schiffman HR, Lore R, Passafiume J, Neeb R (1970) Role of vibrissae for depth perception in the rat (Rattus norvegicus). Anim Behav 18:290-292.

Schoenbaum G, Eichenbaum H (1995) Information coding in the rodent prefrontal cortex. II. Ensemble activity in orbitofrontal cortex. J Neurophysiol 74:751-762.

Shadlen MN, Newsome WT (1998) The variable discharge of cortical neurons: implications for connectivity, computation, and information coding. J Neurosci 18:3870-3896.

Sheth BR, Moore CI, Sur M (1998) Temporal modulation of spatial borders in rat barrel cortex. J Neurophysiol 79:464-470.

Simons DJ (1978) Response properties of vibrissa units in rat SI somatosensory neocortex. J Neurophysiol 41:798-820.

Simons DJ, Carvell GE (1989) Thalamocortical response transformation in the rat vibrissa/barrel system. J Neurophysiol 61:311-330.

Tabachnick BG, Fidell LS (1996) Using multivariate statistics. Redding, MA: Addison-Wesley.

Van der Loos H (1976) Barreloids in mouse somatosensory thalamus. Neurosci Lett 2:1-6.

Victor JD, Purpura KP (1996) Nature and precision of temporal coding in visual cortex: a metric-space analysis. J Neurophysiol 76:1310-1326.

Vincent SB (1912) The function of the vibrissae in the behavior of the white rat. Behav Monogr 1:1-82.

Wilson MA, McNaughton BL (1994) Reactivation of hippocampal ensemble memories during sleep. Science 265:676-679.

Woolsey TA, Van der Loos H (1970) The structural organization of layer IV in the somatosensory region (SI) of mouse cerebral cortex: the description of a cortical field composed of discrete cytoarchitectonic units. Brain Res 17:205-242.

Zhu JJ, Connors BW (1999) Intrinsic firing patterns and whisker-evoked synaptic responses of neurons in the rat barrel cortex. J Neurophysiol 81:1171-1183. 